\title{
Reconstruction of road defects and road roughness classification using vehicle responses with artificial neural networks simulation
}

\author{
H.M. Ngwangwa ${ }^{1}$, P.S. Heyns* ${ }^{1}$, F.J.J. Labuschagne ${ }^{2}$, and G.K. Kululanga ${ }^{3}$ \\ 1. Dynamic Systems Group, Department of Mechanical and Aeronautical Engineering, University of \\ Pretoria, 0002 Pretoria, South Africa.
}

Tel: +2712 420 2432; Fax: +27123625087

e-mail: stephan.heyns@up.ac.za

2. Intelligent Systems and Traffic Management, Built Environment, CSIR, 0001 Pretoria, South Africa.

3. Department of Civil Engineering, University of Malawi, The Polytechnic, Private Bag 303, Chichiri, Blantyre 3, Malawi.

* corresponding author

\begin{abstract}
The road damage assessment methodology in this paper utilizes an artificial neural network that reconstructs road surface profiles from measured vehicle accelerations. The paper numerically demonstrates the capabilities of such a methodology in the presence of noise, changing vehicle mass, changing vehicle speeds and road defects. In order to avoid crowding out understanding of the methodology, a simple linear pitch plane model is employed. Initially, road profiles from known roughness classes were applied to a physical model to calculate vehicle responses. The calculated responses and road profiles were used to train an artificial neural network. In this way, the network renders corresponding road profiles on the availability of fresh data on model responses. The results show that the road profiles and associated defects can be reconstructed to within a $20 \%$ error at a minimum correlation value of $94 \%$.
\end{abstract}

Keywords: Road damage identification, Bayesian regularized NARX network, Road profile reconstruction, International Organization for Standardization power spectral density (ISO PSD) classification, International Roughness Index (IRI), Linear pitchplane vehicle model. 


\section{INTRODUCTION}

This paper proposes a procedure for utilizing measured responses on a vehicle to reconstruct road profiles and their attendant defects. The study seeks to capitalize on the popularization of vehicle information systems, where sensors are increasingly being mounted on vehicles for assessing vehicle performance and the structural integrity of suspensions. González, O'Brien, Li and Cashell [1] argue that despite major improvements in recent years in the quality of road-profiling equipment, these devices remain generally expensive to purchase, their use with time is inefficient and their operation specialized. They demonstrate numerically the applicability of vehicle acceleration measurements for classifying road roughness.

The procedure proposed here may provide an initial and inexpensive assessment of the uneven condition of the road surface. The assessment could either be used directly for making maintenance decisions, especially where condition-triggered maintenance is employed, or could be utilized as input into more comprehensive road maintenance management systems such as Highway Design and Maintenance (HDM). Most feeder and unpaved courses seem to be particularly in need of such procedures, judging from their relatively poor condition compared to paved courses (Brushett, [2]; Burningham and Stankevich [3]).

Hugo, Heyns, Thompson and Visser [4] note that the management and scheduling of maintenance on mine haul roads have not been widely reported in the literature, and that the management of such roads is often inadequate, leading either to over-maintenance or a failure to recognize significant road deterioration. For this reason, Hugo et al. [4] 
have developed a methodology for reconstructing the road profile, based on system characterization. Although the methodology has been successfully applied to a mine haul road, its reliance on extensive system characterization limits the practical feasibility of the approach. The difficulties associated with the practical implementation of such a methodology may be encountered at three levels, namely the determination of system parameters, the formulation of a mathematical model and the inversion of the system's dynamic model. Experiments are largely used for determining system parameters, and they involve several critical simplifying assumptions. Sometimes the most effective experimental procedure may be inhibited by either the unavailability of appropriate technology or the requirements of the business environment.

In relation to heavy vehicle characterisation, effects of inertia and friction in the suspension components are very important although most numerical simulations do ignore them as a trade-off between model accuracy and computational efficiency. Computational efficiency is critical in cases where new hypotheses are being tested or new concepts are being proved, such as in the present study. However, Cebon [5] reports the disastrous effect of not accounting for inertia when determining the suspension parameters, where the leaf spring hysteresis curves for non-corrected springs exhibited strange compression-expansion behaviour, typical of pneumatic suspensions, at higher frequencies.

A comprehensive mathematical model which could simulate all the motions of the vehicle system would be ideal. However, preparing and processing such a model would require a great deal of time and effort. Furthermore, the process of obtaining road 
profiles from measured vehicle responses requires determining an inverse of the vehicle model. It is therefore often necessary to reduce the models dynamically, so as to avoid excessive computational effort when preparing and processing both the direct and the inverse models. Though artificial neural networks (ANN) may not provide physical insight into the behaviour of the vehicle-road interaction system, it may offer significant advantages by eliminating the need for the characterization and calculation of an inverse model.

Thompson, Visser, Miller and Lowe [6] used ANNs to assist them with recognizing the qualitative defects of specific mine haul roads. Thompson et al. [6] realized there was a need for the ability to analyse, recognize and interpret various forms of the same defect signature and also a need for the use of multi-sensor data to isolate faults from different sources. Kang, Lee and Goo [7] used ANNs merely for classifying the severity of faults on unpaved roads by means of power spectral density (PSD) but did not apply the ANNs to profile reconstruction and defect recognition.

This work demonstrates that it is possible to use ANNs to reconstruct specific road defects and classify overall road damage within bounds of very reasonable accuracy by using the accelerations measured on the vehicle. This, as previously stated, eliminates the requirement for the extensive system characterization used by Hugo et al. [4]. The paper includes evaluations of the widely used road roughness indicator, the International Roughness Index (IRI), merely for correlation purposes. The IRI is an intricate scale represented by statistical values which describe overall roughness levels ranging from airport runways to rough unpaved roads. Therefore the indicator may be 
useful where the engineer is primarily interested in interpreting and comparing the overall properties of road surface roughness (in the road's longitudinal direction), but might be a poor parameter when analysing the effects that depend on the road surface as experienced by the road user (Andren [8]). For example, IRI may not indicate the status of roughness over different ranges of wave numbers.

The article is structured as follows. Section 2 describes the proposed methodology for identifying road damage. Relevant theory is presented in Section 3. The section covers the modelling of a vehicle, the generation of road surface topography and Bayesianregularized ANNs. Section 4 describes the dynamic behaviour of the vehicle used in the study. Section 5 verifies the methodology through analyses and discussions on various scenarios and correlation with the widely used IRI classification. The article is concluded in Section 6.

\section{DESCRIPTION OF PROFILE RECONSTRUCTION METHODOLOGY}

The proposed profile reconstruction methodology is loosely based on a technique developed for mine haul road maintenance by Hugo et al. [4]. However, the methodology proposed here eliminates the difficulties encountered in system characterization, which were mentioned in Section 1, by utilizing a supervised learning ANN to reconstruct the road profiles. The network performs the reconstruction by learning the system input-output behavioural patterns from the vehicle model and utilizing them to map the outputs from fresh but related inputs.

In effect, the network is similar to the inverse model but with the unique advantage that it can handle the non-linearity problems that might be difficult for most inverse models 
calculated from direct linear models. In this study, sprung mass acceleration $\ddot{z}_{s}$, front axle acceleration $\ddot{z}_{u f}$ and driver's upper torso acceleration $\ddot{z}_{1}$, were variously used as network inputs while the road profile $z_{r}(t)=z_{r f}$ was the network target.

Fig. 1 presents the procedure used in this study, which can also be applied to a practical test scenario with some modifications. It comprises the following four main parts: generation of training data, creation and training of the network, validation and testing of the network, and identification of road damage. The following discussion covers these stages with reference to the diagram in Fig. 1 and some mathematical expressions developed later in Section 3.

The neural network training data was obtained from the linear pitch plane vehicle model of Fig. 2 and the displacement PSD road classes of Fig 3. Fig. 3 presents the displacement PSD classes of a road with varying degrees of roughness, from a very good condition in class A to a terribly poor condition in class H (ISO 8608 [9]). In this study, a network was trained for each class using the data generated from its lower and upper bound displacement PSDs, so that a network structure was realized for each of the eight ISO PSD classes. Therefore, during simulation of the network, the algorithm searched for a network structure that returned the minimum mean square error value to be used for reconstructing the road profile. The ISO PSD class to which the network structure belonged, gave the roughness class of the road under test. 
According to the theory developed in Section 3, corresponding roadway inputs on the front and rear tyres, $\mathbf{z}_{\mathbf{r}}=\left\{z_{r f}, z_{r r}\right\}^{T}$ for each of the ISO PSDs shown in Fig. 3 can be calculated, accounting for a specified vehicle wheelbase. In order to account for vehicle vibrations due to extraneous sources, engine/driveline forces $f_{e d}$ and tyre/wheel assembly non-uniformity forces $\mathbf{f}_{\mathbf{w}}=\left\{f_{w f}, f_{w r}\right\}^{T}$ were also applied accordingly as random noise to the linear pitch-plane (LPP) model (Fig. 2).

The neural network was created using MATLAB's newnarxsp.m in the Neural Networks Toolbox (Mathworks Inc. [10]). This function creates a series-parallel architecture which feeds back the true output rather than the estimated output. This has two advantages: firstly, the input to the feed-forward network is more accurate; secondly, the resulting network has a purely feed-forward architecture and static backpropagation can be used for training the network (Mathworks Inc., [10]). So the network target $z_{r}(t)$ was fed back via tapped delay lines to form part of network inputs $\mathbf{u}(t)=\left\{\ddot{z}_{s}, \ddot{z}_{u f}, \ddot{z}_{1}\right\}^{T}$ as shown in Fig. 4. The time lags between network inputs and outputs were modelled by allowing for 15 delays on both $\mathbf{u}(t)$ and $z_{r}(t)$. The network was trained by the trainbr algorithm discussed in Section 3.

ISO PSDs different from the ones used in generating the training data were generated and their corresponding road profiles calculated. These were used for validating and testing the network. The network was subsequently simulated and the output of the network $\tilde{z}_{r}(t)$, was plotted over the actual profile $z_{r}(t)$, to validate the network. Then 
road defects were created by adding sinusoidal functions to the actual profiles. The defects were altered by adding simple changes in geometries to account for increase in damage. The mean square errors and correlation coefficients were used in determining the performance of the network, and they are reported in Section 5. The network showed that it never memorized the relationships because it did not over-fit the data but was able to reproduce all the prominent curves in the profiles.

The identification process was performed in two stages. First the network outputs (reconstructed profiles) were matched with the actual road profiles (targets). Secondly, PSDs were calculated using MATLAB's spectrum.welch.m function (Mathworks Inc. [10]). The function used the Hamming window with a segment length of 256 points. The resulting PSDs were then plotted over the ISO PSD classification scale shown in Fig. 3.

\section{THEORY}

\subsection{Vehicle modelling}

For demonstrating the concept proposed in this work, the entire system was modelled by the linear pitch-plane eight-degree of freedom (8DOF) model comprising 4DOFs associated with the vehicle, $1 \mathrm{DOF}$ associated with the driver seat and a further 3DOFs for the seated driver. The linear pitch-plane (LPP) model was chosen because bounce and pitch motions are more dominant than roll motion at all frequencies. For most vehicles, resonances in roll occur at lower frequencies (typically between 0.5 and $1 \mathrm{~Hz}$ ) than resonances in bounce (Gillespie [11]). However, at these low frequencies the PSD ratio of roll displacement input to vertical excitation from the roadway is usually below 
0.1. At high frequencies the PSD ratio approaches unity because the left and right wheel tracks tend to become more uncorrelated. However the vehicle is less responsive to roll input at these frequencies.

The driver seat mass is combined with the mass of the seated driver's lower torso, into a single mass $m_{0}$ in the resulting equation of motion. The seat's foam cushion is modelled by a spring and a damping constant, $k_{0}$ and $c_{0}$ (Fig. 2) whose values are dependent upon static pre-loads (Wei and Griffin [12]). Table 1 shows the characteristics of a 1DOF model of the seat with preload values from 300 to $800 \mathrm{~N}$.

The 3DOF seated-driver model is based on ISO 5982 [13] which does not attach any direct relationships to the body segments. The main advantage of this model is its relative simplicity and linearity, while taking into account human body mass as a parameter of the model (Stein and Múčka [14]). The mass of the seated driver was estimated by the standard assumption that approximately three-quarters of the total seated person's mass acts on the vehicle cabin floor via the seat and the rest acts via the legs and possibly the backrest (Stein and Múčka [14]).

The vibrations are small for most heavy-vehicle driving conditions, so the geometry can be assumed to be linear (Cebon [5]). The equation of motion of a linear vehicle model (with linear geometry and linear springs and dampers representing tyre and suspension elements) can be written in matrix form as (Gillespie [11]) 
$\mathbf{M} \ddot{\mathbf{z}}+\mathbf{C} \dot{\mathbf{z}}+\mathbf{K z}=\mathbf{f}(t)$

where $\mathbf{M}$ is a matrix representing vehicle body mass, $\mathbf{C}$ is a matrix of vehicle damping, $\mathbf{K}$ is the stiffness matrix of the vehicle, $\mathbf{z}$ is a vector of vehicle dynamic responses and $\mathbf{f}(t)$ is a vector of force inputs acting on the vehicle. It was assumed that the pitch angle was so small that kinematical motions at the front axle, rear axle, engine mount and driver's seat might be approximated by the following equations, respectively

$$
\begin{aligned}
& z_{s f}=z_{s}-a_{f} \theta_{s} \\
& z_{s r}=z_{s}+a_{r} \theta_{s} \\
& z_{s e d}=z_{s}-d_{e d} \theta_{s} \\
& z_{s d}=z_{s}-d_{r} \theta_{s}
\end{aligned}
$$

Eq. (1) was applied to each DOF in Fig. 2 and the respective relationships appear in the Appendix to this paper. The property matrices $\mathbf{M}, \mathbf{C}$ and $\mathbf{K}$ are represented by Eqs. (A9) to (A11). Then Eq. (1) was recast into multivariable state space equations given by

$$
\dot{\mathbf{Z}}=\left\{\begin{array}{c}
\hat{\mathbf{0}} \\
\mathbf{M}^{-1} \mathbf{f}
\end{array}\right\}+\left[\begin{array}{cc}
\mathbf{0} & \mathbf{I} \\
-\mathbf{M}^{-1} \mathbf{K} & -\mathbf{M}^{-1} \mathbf{C}
\end{array}\right] \mathbf{Z}
$$

where $\mathbf{I}$ and $\mathbf{0}$ represent identity and null matrices with similar dimensions as the property matrices, $\hat{\mathbf{0}}$ is a null column vector with a length similar to the forcing vector f and $\mathbf{Z}$ is a vector containing the states (displacement and velocity vectors). Eq. (6) was solved using a Runge-Kutta fourth-order integration routine in MATLAB 7. 


\subsection{Road surface topography}

The ISO-proposed road roughness classification is based on the PSDs of road profiles (ISO 8608 [9]). The classification identifies eight road roughness levels ranging from class $\mathrm{A}$ to class $\mathrm{H}$ in increasing order of roughness, where the first five of these classes (A to E) are really important in practice. In the ISO classification, the relationships between the displacement power spectral density $S_{u}(\kappa)$ and the wave number $\kappa$ for different classes of road roughness may be approximated by (ISO 8608 [9])

$$
S_{u}(\kappa)= \begin{cases}S_{u}\left(\kappa_{0}\right)\left(\frac{\kappa}{\kappa_{0}}\right)^{-n_{1}} & \frac{\kappa}{\kappa_{0}} \leq 1 \\ S_{u}\left(\kappa_{0}\right)\left(\frac{\kappa}{\kappa_{0}}\right)^{-n_{2}} & \frac{\kappa}{\kappa_{0}}>1\end{cases}
$$

where $S_{u}(\kappa)$ is expressed in $\mathrm{m}^{3}$ cycle $^{-1}, \kappa$ is expressed in cycles $\mathrm{m}^{-1}, S_{u}\left(\kappa_{0}\right)$ is the displacement power spectral density at the datum or cut-off wave number $\kappa_{0}$, which is equal to $1 / 2 \pi$ cycles $^{-1}$. With the constants $n_{1}$ and $n_{2}$ equal to 2.0 and 1.5 respectively (ISO 8608 [9]), Eq. (7) can be used to generate plots of $S_{u}(\kappa)$ as a function of the wave number ratio $\kappa / \kappa_{0}$ as shown in Fig 3. This expresses a mathematical fit to empirical data, based on extensive measurements of road profiles on European roads (La Barre, Forbes and Andrews [15]). Cebon [5] and Wong [16] recommend different values for the constants, based on earlier ISO standards. Dodds and Robson [17] also specify different ranges of exponents depending on the class of the road. This is not critical, however, in the context of the current study which focused on developing the overall methodology for assessing road damage. 
The displacement power spectral density as given in Eq. (7) is usually calculated from the measurement of surface roughness described by vertical ordinates at equally spaced points along the road. However, in the absence of such measurements, pseudo-random profiles can be generated to fit those spectral densities. Cebon [5] presents a formula for generating a one-dimensional random profile as:

$z_{r}=\sum_{k=0}^{N-1} \sqrt{S_{k}} e^{i\left(\theta_{k}+\frac{2 \pi k r}{N}\right)}, \quad r=0,1,2, \ldots(N-1)$

where $S_{k}=(2 \pi / N \Delta) S_{11}(\gamma k)$ and $S_{11}(\gamma k)$ is the target spectral density,

$\gamma k=2 \pi k / N \Delta$ represents wave number in $\mathrm{rad} / \mathrm{m}, \Delta$ is the distance interval between successive ordinates of the surface profile, and $\theta_{k}$ is a set of independent random phase angles uniformly distributed between 0 and $2 \pi$.

The road inputs to the vehicle considered here cover a range of wave number ratios between 0.01 and 10 . The frequency $(\mathrm{Hz})$ is related to the wave number ( cycles $/ \mathrm{m}$ ) by the relationship $f=v \kappa$, where $v$ is the vehicle velocity in $\mathrm{m} / \mathrm{s}$. Therefore the vehicle speeds between 20 and $80 \mathrm{~km} / \mathrm{h}$ considered in this study, span different frequency ranges between 0.009 and $35.4 \mathrm{~Hz}$. This range is sufficient to excite the important frequencies of the vehicle under study.

Road profiles can be determined by using Eqs. (7) and (8) in the following manner: Initially the road roughness is specified by a value of the constant $S_{u}\left(\kappa_{0}\right)$ chosen from the ISO standard representing a particular road roughness classification, after which Eq. 
(7) can be applied to compute a displacement PSD $S_{u}(\kappa)$. The resulting PSD is subsequently converted from an implicit function of $\kappa$ (wave number in cycles $/ \mathrm{m}$ ) into an implicit function of $\gamma(\mathrm{rad} / \mathrm{m})$ to determine $S_{k}$ which is passed on to Eq. (8) to calculate the corresponding road profile.

\subsection{Bayesian-regularized NARX neural network}

Artificial neural networks are composed of simple elements (neurons) operating in parallel. The networks typically operate by adjusting the values of the connections (weights) between the neurons so that particular inputs lead to specific target outputs. Recently ANN type models have been popularly used to model non-linear dynamics due to their high adaptability to various non-linear systems, the ready availability of tools and the proliferation of computer algorithms (Wong and Worden [18]). This paper describes how the ANN was trained to perform online identification of the vehicle-road interaction system so that road profiles could be reconstructed from given sets of data acquired from the vehicle. Although the vehicle model used in this study was linear, the parameters of interest are not related in a simple linear manner. It would therefore be inappropriate to use linear system identification models.

One of the popular networks for non-linear function approximation is the Nonlinear AutoRegressive with Exogenous Inputs (NARX) network (Wong and Worden [18]). Wong and Worden [18] report that the NARX network's underlying universal approximation theory guarantees that a basic 3-layer multilayer perceptron (MLP) can perform input-output mapping for any continuous function. It computes the current output using an MLP that takes as input a series of past system input $\mathbf{u}(t-i)$ and past 
output values $z_{r}(t-i)$. The NARX network's operation is described by Mathworks Inc $[10]$

$\tilde{z}_{r}(t)=\mathbf{g}\left(\mathbf{u}\left(t-n_{u}\right), \ldots, \mathbf{u}(t-1), \mathbf{u}(t), z_{r}\left(t-n_{z}\right), \ldots, z_{r}(t-1)\right)$

where $\mathbf{g}()$ is a non-linear mapping function of the MLP, where $\mathbf{u}(t)$ is a vector containing the acceleration input sequences and $z_{r}(t)$ is the target at time $t$, and $n_{u}$ and $n_{z}$ are the maximum input and output lags respectively. The architecture of the NARX network is shown in Fig. 4. The series-parallel architecture was adapted to reduce the computational cost, since it has no feedback loop inside the model itself and employs static backpropagation for the adjustment of parameters (weights). The vehicle-driver system is assumed to be stable within the limits prescribed by the space of the adjusted weights and biases (parameters), implying that the systems have bounded inputs and bounded outputs.

The network was trained by a Levenberg-Marquardt algorithm which computes the new weights $w_{n e w}$ via the relationship (Mathworks Inc. [10]; Hagan and Menhaj [19]; Bishop [20])

$$
\mathbf{w}_{\text {new }}=\mathbf{w}_{\text {old }}+\left(\mathbf{J}^{T} \mathbf{J}+\lambda \mathbf{I}\right)^{-1} \mathbf{J}^{T} \boldsymbol{\varepsilon}\left(\mathbf{w}_{\text {old }}\right)
$$

where $\mathbf{I}$ is the identity matrix, $\varepsilon\left(w_{\text {old }}\right)$ is an error vector at the current point, $\mathbf{J}$ is a Jacobian matrix (typically consisting of first partial derivatives of the error with respect to the parameters) and $\lambda$ is a parameter governing the step size. This value is expected to vary during the minimization process. The Levenberg-Marquardt algorithm was chosen because of its good performance in function approximation and its computational 
efficiency based on the fact that it avoids the more costly evaluation of the Hessian matrix (Mathworks Inc. [10]).

To enable the network to perform as well on novel inputs as on training set inputs, the NARX network utilized Bayesian regularization. Typical training only involves reducing the sum of squared errors, whereas regularization includes minimizing the sum of squares of the network weights to achieve optimal network performance. Therefore the objective function in regularization is written as

$\boldsymbol{\varepsilon}=\boldsymbol{\beta} \boldsymbol{\varepsilon}_{D}+\boldsymbol{\alpha} \boldsymbol{\varepsilon}_{W}$

where $\boldsymbol{\varepsilon}_{D}$ is the sum of squared errors, $\boldsymbol{\varepsilon}_{W}$ is the sum of squares of the network weights, and $\boldsymbol{\alpha}$ and $\boldsymbol{\beta}$ are parameters of the objective function (Hagan and Menhaj [19]; Bishop [20]), compromising between fitting the data and producing a smooth network response. In Bayesian regularization this is performed by searching for the optimal weight that maximizes the posterior probability which is equivalent to minimizing the regularized objective function (Bishop [20]).

\section{DESCRIPTION OF THE VEHICLE DYNAMICS}

The linear pitch-plane (LPP) model of Fig. 2 is now used to numerically demonstrate the procedure for reconstructing the road profiles, using ANN simulation. The model takes into account pitch motion which forms an important part of overall heavy-vehicle vertical vibratory response during normal road operation (Gillespie [11]). In this evaluation, road profiles were synthetically generated from known cases of road damage that were determined within the ISO roughness classifications (Fig. 3). 
An LPP model of the haul truck which had been characterised by Hugo [21] was developed. Hugo [21] determined the tyre characteristics in situ whereas those for the hydro-pneumatic struts were determined by fitting the ideal gas law and the energy equation to experimental observations on the nitrogen and oil in the struts. Tyre damping $c_{t}$ was assumed to be negligible. Although the struts exhibited non-linear characteristics, they were conveniently linearized around their equilibrium states. Table 2 shows the model parameters used in the LPP model.

The distances of the front $a_{f}$ and rear $a_{r}$ axles from the centre of gravity (cog) of the vehicle were 3.7 and $1.8 \mathrm{~m}$ respectively. The driver's seat was located $0.2 \mathrm{~m}$ away from the vehicle's cog towards the front axle. The engine and driveline forces were assumed to act at $2.5 \mathrm{~m}$ away from the cog on the front axle side. Tables 1 and 3 show the model parameters, assuming that the total standing mass of the driver was $70 \mathrm{~kg}$.

When the models were subjected to unit step road inputs, the sprung mass responses exhibited fading sinusoidal vibrations at the frequency of $4.1 \mathrm{~Hz}$, corresponding to axle hop resonance, and at $0.95 \mathrm{~Hz}$, corresponding to the sprung mass bounce resonance (Figs. 5(a) and (b)). The first sinusoidal motion dominated the vibration of the vehicle immediately after both axles had passed the step and died out within a short while after that. The second part of the motion persisted much longer.

Figs. 5(a) and (b) furthermore show that the rear suspension deflection was in phase and vibrated at the same frequency with the vehicle body vibration, while the front suspension deflection was at a slightly higher frequency being dominated by pitch 
motion at a frequency of $1.4 \mathrm{~Hz}$. This was expected since the moment arm of the front suspension from the cog was more than twice as long as that for the rear suspension. The influence of pitch motion on the vibration of the truck is represented by the differences between quarter-car (QC) model results (Figs. 5(a) and (c)) and LPP model results (Figs. 5(b) and (d)). The differences in the phases and magnitudes of displacements between the two model results are significant and justify the preference for the LPP model.

\section{NUMERICAL STUDIES}

The proposed methodology is demonstrated for different vehicle speeds for cases of generally varying roughness grades, emerging and growing defects and for different vehicle payloads and various levels of noise. In all these cases the NARX network was composed of twenty neurons with tangent sigmoid (tansig) transfer functions in the hidden layer and one neuron having a linear (purelin) transfer function in the output layer. The network used a training function trainbr which updates the weights and biases according to the Levenberg-Marquardt optimization (Eq. (10)) by minimizing a combination of squared errors and weights (Eq. (11)) in order to obtain a combination enabling the network to generalize well (Mathworks Inc. [10]; Bishop [20]).

The computational efficiencies of the network for various combinations of inputs and delays were computed and are presented in Table 4. The results show that the differences in errors and correlations were influenced more by the number of input delays than by the number of network inputs. Though the sprung mass acceleration $\ddot{z}_{s}$ with 8 delays shows the best performance, it exhibited too much inflexibility in the presence of road surface imperfections, like speed humps or potholes. The use of front 
unsprung mass acceleration $\ddot{z}_{u f}$ with 15 delays might provide an alternative efficient solution computationally, but the practical difficulties of mounting accelerometers on the vehicle axle frustrate its application. Hence the sprung mass acceleration $\ddot{z}_{s}$ with 15 delays was chosen owing to its relatively better stability, comparative computational efficiency, and ease of practical implementation. So a single-input single-output, 1-20-1 NARX network was created, which had to yield road profile, $\tilde{z}_{r}(t)=z_{r f}$ as its output. It is envisaged however that the use of the sprung mass acceleration as sole network inputs might not be as efficient in a practical test situation, owing to the existence of so many influential features which could better be represented by complementing the sprung mass acceleration with other responses.

The length of the road under study was $512 \mathrm{~m}$ and was sampled similarly for all velocities at 4 samples per $\mathrm{m}$. The results include evaluations of the correlation coefficient R and the error $\varepsilon$. The correlation coefficient was calculated by using MATLAB's postreg.m function, and the error $\varepsilon$ was computed from the mean square error values between the reconstructed and actual profiles using MATLAB's mse.m function. The correlation coefficient indicates how well the network's reconstructed profile matches the actual road topography, whereas the error measures the accuracy of fit between the two profiles.

The following sections contain an investigation of various case studies of interest under testing situations. Section 5.1 addresses the general problem of reconstructing a road profile of any roughness class and the ability to identify its class within the ISO PSD classification scale in Fig. 3. Section 5.2 deals with the problem of reconstructing and 
identifying emerging defects over the road surface. The problems of applying the methodology under changing or growing defects are presented in Section 5.3. The methodology is applied to different noise levels and vehicle payloads in Sections 5.5 and 5.6 respectively.

\subsection{General road roughness identification}

The aim of this case study was to show whether the methodology could be applied to identifying roads that typically fell into different roughness classes shown in Fig. 3. Four roads belonging to classes A, C, D and F were arbitrarily chosen and generated using Eqs. (7) and (8) as outlined in Section 2. The resulting profiles were applied to the vehicle model to determine the responses. The vehicle responses were then applied to the NARX network previously trained with road profiles lying on class boundaries to reconstruct the corresponding road profiles.

Fig. 6 plots the actual profiles over reconstructed profiles for the four test roads. The figure shows that actual and reconstructed profiles correlated uniformly at $98.1 \%$ with a reconstruction error around $10 \%$ attaining a maximum of $11 \%$ for test profile $\mathrm{F}$. These network performance indicators (correlation coefficients and error percentages) were averaged over a set of ten tests. The errors resulted from network failure to fit corners in the road profiles perfectly. In a previous study (Ngwangwa, Heyns, Labuschagne and Kululanga [22]) it was noted that the errors were also influenced by the distance between the displacement PSD of the profiles used during network training and those used in the test. These errors can be reduced by generating more training profiles between any two class boundaries, however, that causes network training to become onerous. Alternatively, training with Gaussian noise at different levels of noise was 
shown to improve network performance although that resulted in increasing the roughness class and required some sort of correction for the added noise.

In Fig. 7, the reconstructed profiles were correctly classified into their respective roughness grades $\mathrm{A}, \mathrm{C}, \mathrm{D}$ and $\mathrm{F}$ as can be observed from their good correspondence with the actual PSDs (dotted line). These roughness classes are actually interpreted on the ISO PSD scale as very good, average, poor and extremely poor respectively. IRI scale (Sayers and Karamihas [24]) identifies the same test roads by the values 2.5, 10.0, 19.9, and 79.8 representing the regions covered by new pavements, maintained unpaved roads or damaged pavements with frequent shallow depressions some deep, rough unpaved roads with erosion gulleys and deep depressions. The test road corresponding to ISO PSD class F or IRI value of 79.8 was used here merely for theoretical purposes because it lies outside the practical limits of allowable road severity.

\subsection{Defect identification under different speeds}

The purpose of this case study was to show the ability of the methodology in identifying localized surface irregularities in the longitudinal direction of the road at different vehicle speeds. The pothole had a width of $2 \mathrm{~m}$ and was located along the road at a distance of $50 \mathrm{~m}$ from the start. It had an amplitude of $0.012 \mathrm{~m}$ expressed as a sinusoidal function of distance $x$, so that the defect profile can be given by

$z_{\text {def }}=\left\{\begin{array}{cc}0.012 \sin \frac{2 \pi x}{\lambda} & x \geq x_{1} \text { and } x \leq x_{2} \\ 0 & \text { otherwise }\end{array}\right.$ 
where $x$ represents the distance along the test road, $\lambda$ is the defect wavelength, and $z_{\text {def }}$ is the profile's vertical ordinate at distance $x$. Therefore the road profile to be reconstructed was given by the summation of Eqs. (8) and (12).

$z_{r x}=z_{r}+z_{d e f}$

However, the use of PSD classification restricted the defects, making them periodic in nature. The identification of the pothole was examined for the vehicle speeds of 20, 30 , 50 and $80 \mathrm{~km} \mathrm{~h}^{-1}$. Figs. 8(a), (b), (c) and (d) show results of reconstructing the same pothole when the vehicle traverses it at the speeds given above. The correlation values and error percentages on top of the graphs represent network performance over the entire road length.

The pothole was correctly located between 50 and $52 \mathrm{~m}$ at all vehicle speeds. The minor shift of $0.2 \mathrm{~m}$ in the reconstructed profile might have been caused by an inability to model network delays perfectly, given the inefficiencies of a trial and error procedure. However, it may be argued that for a heavy vehicle travelling at speeds above $20 \mathrm{~km} \mathrm{~h}^{-1}$, such a small delay, corresponding to a maximum time delay of $0.036 \mathrm{~s}$, may be insignificant. Besides, the benefits obtained by achieving a zero delay fit by a trial and error procedure could not justify the time and effort required to achieve such a goal. Figs. 9 and 10 show the profile reconstructions and classifications for three different damage scenarios at each of the specified vehicle speeds. The different damage scenarios are applied to a class A road. The results in Fig. 9 show very good correspondence between the actual and reconstructed profiles with a bit of random performance around some of the sharp turns, in that the reconstructed profiles either overshoot or smoothen out. This network behaviour makes it difficult to distinguish 
among different degrees of roughness within any particular roughness class. In Fig.10, for example, it can be observed that for the same underlying roughness, the road class either lies close to the lower or upper boundary of class A. The higher PSDs between wave number ratios of 0.4 and 1.1 correspond to surface defects with various wavelengths. IRI classification shows that the test roads are typically new pavements but fails to reflect the small surface imperfections.

\subsection{Identification under changing/growing defects}

In this case study, the underlying rationale was to show the neural network's capabilities under changing/growing defects. The defect changes are represented in terms of damage scenarios Dam. $\# N_{i}$, where $N_{i}$ denotes 1, 2, 3, and 4. These damage scenarios are distinguished by the lengths of deteriorated road sections and assumed wavelengths of the defects. Fig. 11 shows that the reconstructed profiles are above $96 \%$ level of correlation with errors under $20 \%$ for all the damage scenarios. In both graphs (a) and (b) the dash-dotted lines trend average network performance taken over the four different vehicle speeds.

Fig.12 shows average network performance over all the damage scenarios at each vehicle speed. The profiles are reconstructed at an average correlation level of above $97 \%$ with errors lying between 10 and $11 \%$. These results show that the level of damage does not necessarily have any significant influence on network performance at any given vehicle speed. Errors resulted from the usual network shortcomings as previously pointed out and the difficulties encountered in accurately estimating the necessary number of delays in the feedback loop of the NARX network. 
Fig. 13 shows the displacement PSDs with the corresponding IRI values in $\mathrm{m}$ per $\mathrm{km}$. The general level in the values of IRI indicates that the road is in very good condition and in terms of the condition of pavements; it implies that the pavement lies between new pavements to older pavements (Sayers and Karamihas [24]). This interpretation agrees with the classification using the ISO PSDs where the road roughness, especially at higher wave numbers, belongs to class A.

The ISO PSDs show that for Dam. \#1 the road lies in class A at lower wave numbers and slightly under class A at very high wave numbers. Dam. \#2 largely lies within class A, though at lower wave numbers, it lies very close to its upper boundary. In Dams. \#3 and \#4 the displacement PSD lies in class $\mathrm{C}$ around a wave number ratio of 0.6.

However, that information is so obscure in the IRI classifications of 2.36 and 2.41 , both of which may typically be interpreted as new pavements having near-perfect conditions though not absolutely perfect. This implies that the surface irregularities have to grow beyond these levels in order for the IRI classification to signal emergence of surface imperfections that need attention. Typically IRI values in excess 3.5 may be considered significant.

The analysis helps to underscore the advantage of ISO PSD classification over IRI classification. The only shortcoming of the ISO PSD classification being that the road defects should be estimated as periodic functions. The more interesting result in this analysis consists in that both classifications agree on the underlying roughness class. Thus it is possible to use the two methods simultaneously to enhance more understanding of the nature of road damage. 


\subsection{Identification under noisy conditions}

In this case study, Band-Limited White (BLW) Noise was applied to a class A road having a surface irregularity. The added BLW Noise lay in the frequency range $1-25$ $\mathrm{Hz}$ with signal-noise ratios (SNR) between 0 and 0.5 . Fig. 14 shows that the error in reconstructing the road surface profiles grows with noise level and it might be necessary for practical purposes to keep the SNR below 0.2. Fig. 15 shows ISO PSD and IRI classifications at $0.2 \mathrm{SNR}$ level for different vehicle speeds. IRI classifies the road around an average roughness level of 2.5 indicative of new pavements without surface imperfections, although the picture is slightly different at the lowest vehicle speed of 20 $\mathrm{km} / \mathrm{h}$ where it approaches a domain of new pavements with surface defects. ISO PSD lines largely lie within class A with slight upward shift in lower wave numbers which are emphasized around the wave number ratios $0.4-1.1$ corresponding to the surface defects' wavelengths.

It is further noted that profiles with smaller SNR are well classified into class A whereas those with larger SNR tend to shift upwards into higher roughness classes particularly in lower wave numbers. Theoretically, that is expected because small levels of noise make the NARX network behave like a regularised network. Bishop [20] notes that during training, provided the noise amplitude is small, so that the neglect of the higher-order terms in the Taylor expansion is valid, the minimization of the sum-of-squares error with noise added to the input data is equivalent to the minimization of the regularized sum-of-squares error without the addition of noise. 


\subsection{Identification under different vehicle weight}

The gross weights of vehicles vary significantly during operation owing to changes in payloads. It is assumed that suspension characteristics remain generally invariant over a larger proportion of the vehicle's operational period. Therefore this case study examines how the methodology performs under vehicle weight changes for different speeds. The weights are changed by adding to the gross vehicle weight (GVW) proportions of its own weight between 0 and 0.5 . The vehicle was assumed to operate along a lowerboundary class $\mathrm{E}$ rough road at a speed of $20 \mathrm{~km} \mathrm{~h}^{-1}$.

The results in Fig.16 show that profiles are accurately reconstructed with errors around $20 \%$ at greater than $94 \%$ correlation level. The road roughness is also accurately classified in terms of both ISO PSD and IRI classifications as shown in Fig. 17. The IRI classification of 22.66 implies a rough unpaved road which corresponds to ISO PSD's lower-boundary E road. The results show that changes in vehicle weight do not significantly affect the performance of the network, at least within practical limits of payload variation.

\section{CONCLUSIONS}

This work was aimed at numerically demonstrating a methodology for classifying road damage based on road profile reconstruction and using artificial neural networks. The use of neural networks is believed to offer two main advantages. The primary advantage is that it does not require excessive system characterization. All it requires is the road profile data representative of various degrees of road roughness as network targets, and accelerations measured on the vehicle system as network inputs. In this particular study, the use of accelerations other than those measured on the sprung mass offered no 
significant advantages. However, determining the optimal number of network inputs might be application-specific and is a subject of further study in this work.

The second advantage of the neural network based methodology is that it requires relatively fewer analytical skills to create the network than the parametric model. The current developments in technical computing have ensured that most neural network models become standard and implemented in technical software e.g. MATLAB, whereas the development of physical parametric models still demands a great deal of technical skills, even in virtual-computing environments. Besides, the physical parametric models require the calculation of the inverse models in order to determine the road profiles from measured vehicle accelerations, which may also be very rigorous on their own.

The performance of the methodology has been demonstrated for varying roughness grades, emerging surface defects, and changing or growing surface defects under varying conditions of noise, vehicle payload and speed through evaluations of correlation coefficients and mean square errors. The network performance does not exhibit any clear dependencies on the different conditions under study except that under harsh noisy conditions, the performance deteriorated significantly.

The methodology was observed to correlate well with IRI which is a widely used roughness indicator. The study reinforces the combined use of IRI and ISO PSD classification to enhance road surface condition interpretation. More promising is the fact that a practical test case inherently includes the filtering effects of tyre 
envelopment, which were not considered in this numerical study. Therefore some of the requirements for stringent accuracy in this numerical study might be superfluous in a practical situation. Accordingly the proposed methodology aptly provides a methodology for approximating and making reliable initial judgements of road surface damage.

\section{ACKNOWLEDGEMENTS}

The support of the Council for Scientific and Industrial Research (CSIR) and the National Research Foundation under the South African Co-operation Fund for Scientific Research and Technological Developments, is gratefully acknowledged.

\section{APPENDIX}

The dynamic equations of motion for the system in Fig. 2 may be derived by considering the free-body diagrams. The system has a total of 8 DOFs comprising: sprung mass vertical displacement $z_{s}$, sprung mass pitch $\theta_{s}$, front axle vertical displacement $z_{u f}$, rear axle vertical displacement $z_{u r}$, seat displacement $z_{0}$, and displacements on the driver's body $\left(z_{1}, z_{2}, z_{3}\right)$. In the following, dynamic equations are formulated for each mass.

The formulation of sprung mass motions requires the kinematic relations in Eqs. (2) to (5) presented under Section 3. So the dynamics of the sprung mass can be written as

$$
\begin{aligned}
& M_{s} \ddot{z}_{s}+k_{s f}\left(z_{s f}-z_{u f}\right)+c_{s f}\left(\dot{z}_{s f}-\dot{z}_{u f}\right)+k_{s r}\left(z_{s r}-z_{u r}\right)+ \\
& c_{s r}\left(\dot{z}_{s r}-\dot{z}_{u r}\right)+k_{0}\left(z_{s d}-z_{0}\right)+c_{0}\left(\dot{z}_{s d}-\dot{z}_{0}\right)=f_{e d} \\
& I_{s} \ddot{\theta}_{s}-a_{f} k_{s f}\left(z_{s f}-z_{u f}\right)-a_{f} c_{s f}\left(\dot{z}_{s f}-\dot{z}_{u f}\right)+a_{r} k_{s r}\left(z_{s r}-z_{u r}\right)+ \\
& a_{r} c_{s r}\left(\dot{z}_{s r}-\dot{z}_{u r}\right)-d_{r} k_{0}\left(z_{s d}-z_{0}\right)-d_{r} c_{0}\left(\dot{z}_{s d}-\dot{z}_{0}\right)-d_{e d} f_{e d}
\end{aligned}
$$


For the front and rear axles, the equations of motion are

$$
\begin{aligned}
& m_{u f} \ddot{z}_{u f}+k_{s f}\left(z_{u f}-z_{s f}\right)+c_{s f}\left(\dot{z}_{u f}-\dot{z}_{s f}\right)+ \\
& k_{t f}\left(z_{u f}-z_{r f}\right)+c_{t f}\left(\dot{z}_{u f}-\dot{z}_{r f}\right)=f_{w f} \\
& m_{u r} \ddot{z}_{u r}+k_{s r}\left(z_{u r}-z_{s r}\right)+c_{s r}\left(\dot{z}_{u r}-\dot{z}_{s r}\right)+ \\
& k_{t r}\left(z_{u r}-z_{r r}\right)+c_{t f}\left(\dot{z}_{u r}-\dot{z}_{r r}\right)=f_{w r}
\end{aligned}
$$

The equations for the seat and driver are

$$
\begin{aligned}
& m_{0} \ddot{z}_{0}+k_{0}\left(z_{0}-z_{s d}\right)+c_{0}\left(\dot{z}_{0}-\dot{z}_{s d}\right)+k_{1}\left(z_{0}-z_{1}\right)+ \\
& c_{1}\left(\dot{z}_{0}-\dot{z}_{1}\right)+k_{3}\left(z_{0}-z_{3}\right)+c_{3}\left(\dot{z}_{0}-\dot{z}_{3}\right)=0 \\
& m_{1} \ddot{z}_{1}+k_{1}\left(z_{1}-z_{0}\right)+c_{1}\left(\dot{z}_{1}-\dot{z}_{0}\right)+k_{2}\left(z_{1}-z_{2}\right)+c_{2}\left(\dot{z}_{1}-\dot{z}_{2}\right)=0 \\
& m_{2} \ddot{z}_{2}+k_{2}\left(z_{2}-z_{1}\right)+c_{2}\left(\dot{z}_{2}-\dot{z}_{1}\right)=0 \\
& m_{3} \ddot{z}_{3}+k_{3}\left(z_{3}-z_{0}\right)+c_{3}\left(\dot{z}_{3}-\dot{z}_{0}\right)=0
\end{aligned}
$$

Upon making the necessary substitutions for the kinematic equations in the relevant equations above, and grouping like terms according to degrees of freedoms and their derivatives, the above equations can be rewritten in the compact matrix form of Eq. (1), where the property matrices and force vector are given by Eqs. (A9) to (A11).

$$
\mathbf{M}=\operatorname{diag}\left(\left[\begin{array}{llllllll}
M_{s} & I_{s} & m_{u f} & m_{u r} & m_{0} & m_{1} & m_{2} & m_{3}
\end{array}\right]\right)
$$




$$
\mathbf{K}=\left[\begin{array}{llllllll}
k_{z s 1} & k_{\theta s 1} & k_{z u f 1} & k_{z u r 1} & k_{z 0,1} & & & \\
k_{z s 2} & k_{\theta s 2} & k_{z u f 2} & k_{z u r 2} & k_{z 0,2} & & & \\
k_{z s 3} & k_{\theta s 3} & k_{z f u 3} & & & & & \\
k_{z s 4} & k_{\theta s 4} & & k_{z u r 4} & & & & \\
k_{z s 5} & k_{\theta s 5} & & & k_{z 0,5} & k_{z 1,5} & & k_{z 3,5} \\
k_{z s 6} & k_{\theta s 6} & & & k_{z 0,6} & k_{z 1,6} & k_{z 2,6} & \\
& & & & & k_{z 1,7} & k_{z 2,7} & \\
& & & & k_{z 0,8} & & & k_{z 3,8}
\end{array}\right]
$$

where all elements with zero values are not indicated and the matrix elements are given by the following relationships:

$$
\begin{aligned}
& k_{z s 1}=k_{s f}+k_{s r}+k_{0}, \quad=k_{\theta s 1} \quad-k_{s f} a_{f}+k_{s r} a_{r}-k_{0} d_{r} \\
& k_{z u f 1}=-k_{s f}, \quad k_{z u r 1} \quad-k_{s r}, \quad k_{z 0,1} \quad-k_{0} \\
& k_{z s 2}=-k_{s f} a_{f}+k_{s r} a_{r}-k_{0} d_{r},=k_{\theta s 2} \quad k_{s f} a_{f}^{2}+k_{s r} a_{r}^{2}+k_{0} d_{r}^{2} \\
& k_{z u f 2}=k_{s f} a_{f}, \quad k_{z u r \overline{2}} \quad-k_{s r} a_{r}, \quad k_{z 0,2}=k_{0} d_{r} \\
& c_{z s 3}=-c_{s f},=c_{\theta s \overline{3}} \overline{3} \quad c_{s f} a_{f}, \quad c_{z u f 3} \quad c_{s f}+c_{t f} \\
& k_{z s 4}=-k_{s r}, \quad k_{\theta s 4} \quad-k_{s r} a_{r}, \quad k_{z u r 4} \quad k_{s r}+k_{t f} \\
& k_{z s 5}=-k_{0},=k_{\theta s \overline{5}} \quad k_{0} d_{r}, \quad k_{z 0,5} \quad k_{0}+k_{1}+k_{3} \\
& k_{z 1,5}=-k_{T}, \quad k_{z 3,5} \quad-k_{3} \\
& k_{z 0,6}=-k_{T}, \quad k_{z 1,6} \quad k_{1}+k_{2}, \quad k_{z 2,6} \quad-k_{2} \\
& k_{z 1,7}=-k_{2}, \quad k_{z 2,7}=k_{2}, k_{z 0,8}=-k_{3}, \quad k_{z 3,8}=k_{3}
\end{aligned}
$$

The corresponding equations for the damping matrix $\mathbf{C}$ and its elements are similar.

$$
\mathbf{f}=\left[\begin{array}{llllllll}
F_{1} & F_{2} & F_{3} & F_{4} & 0 & 0 & 0 & 0
\end{array}\right]^{\mathrm{T}}
$$

where

$$
F_{1}=f_{e d}, \quad F_{2}-d_{e d} f_{e d}, F_{3}=c_{t f} \dot{r}_{r f}+k_{t f} z_{r f}+f_{w f}, \quad F_{4}=c_{t r} \dot{z}_{r r}+k_{t r} z_{r r}+f_{w r}
$$




\section{REFERENCES}

[1] González A, O'Brien E J, Li Y.-Y, Cashell K. The use of vehicle acceleration measurements to estimate road roughness. Vehicle System Dynamics 46:6 (2008) 483499.

[2] Brushett S. Experience in reforms of road maintenance financing and management in sub-Saharan Africa. Transport and Communications Bulletin for Asia and the Pacific 75 (2005) 25-43.

[3] Burningham S, Stankevich N. Why road maintenance is important and how to get it done. Transport Note No TRN-4 (Jun, 2005), World Bank, Washington D.C.

[4] Hugo D, Heyns PS, Thompson RJ, Visser AT. Haul road defect identification and condition assessment using measured truck response, Journal of Terramechanics 45 (2008) $79-88$.

[5] Cebon D. Handbook of vehicle-road interaction, Swets \& Zeitlinger Publishers, Lisse, 1999.

[6] Thompson RJ, Visser AT, Miller RE, Lowe NT. Development of real-time mine road maintenance management system using haul truck and road vibration signature analysis. Transportation Research Record 1819A (2003) 305 - 312.

[7] Kang D.-K, Lee S.-H, Goo S.-H. Development of standardization and management system for the severity of unpaved test courses. Sensors 7 (2007) 2004-2027.

[8] Andrén P. Power spectral density approximations of longitudinal road profiles. International Journal of Vehicle Design $40(1,2,3)$ (2006).

[9] International Organization for Standardization ISO 8608: 1995(E), Mechanical vibration - Road surface profiles - Reporting of measured data, 1995.

[10] Mathworks Inc., MATLAB Help Tutorial, 2007. 
[11] Gillespie TD. Fundamentals of vehicle dynamics. Society of Automotive Engineers, Inc., Warrendale, 1992.

[12] Wei L, Griffin MJ. The prediction of seat transmissibility from measures of seat impedance. Journal of Sound and Vibration 214(1) (1998) 121-137.

[13] International Organization for Standardization ISO 5982:2001, Mechanical Vibration and Shock - Range of idealized values to characterize seated-body biodynamic response under vertical vibration, 2001, Geneva.

[14] Stein GJ, Múčka P. Theoretical investigation of a linear planar model of a passenger car with seated people. Proc. IMechE Part D: J. Automobile Engineering Vol. 217 (2003) $257-268$.

[15] La Barre RP, Forbes RT, Andrews S. The measurement and analysis of road surface roughness. Motor Industry Research Association Report No. 1970/5, 1970.

[16] Wong JY. Theory of ground vehicles. John Wiley \& Sons, Inc., New York, 1993.

[17] Dodds CJ, Robson JD. The description of road surface roughness. Journal of Sound and Vibration 31(2) (1973) 175 - 183.

[18] Wong CX, Worden K. Generalised NARX shunting neural network modelling of friction. Mechanical Systems and Signal Processing 21 (2007) 553-572.

[19] Hagan MT, Menhaj MB. Training feedforward networks with the Marquardt algorithm. IEEE Transactions on Neural Networks 5:6 (1994) 989-993.

[20] Bishop CM. Neural networks for pattern recognition, Oxford University Press, Oxford, 1995.

[21] Hugo D. Haul road defect identification and condition assessment using measured truck response, MEng Thesis, Department of Mechanical and Aeronautical Engineering, University of Pretoria, South Africa, 2005. 
[22] Ngwangwa HM, Heyns PS, Labuschagne FJJ, Kululanga GK. Monitoring of heavy vehicle dynamic response for road condition monitoring: An overview of the web based artificial intelligence technique, Proceedings of the Southern African Transport Conference (SATC2008), 7-10 July 2008, Pretoria.

[23] Sayers MW, Gillespie TD, Paterson WDO. Guidelines for Conducting and Calibrating: Road Roughness Measurements, World Bank Technical Paper Number 46, The World Bank, Washington, D.C., 1986.

[24] Sayers MW, Karamihas SM. The little book of profiling: Basic information about measuring and interpreting road profiles. University of Michigan, 1998. 


\section{TABLE CAPTIONS}

[1] Table 1. Static pre-load dependent parameters of the SDOF cushioned seat (from Wei and Griffin [12])

[2] Table 2. Parameters of QC and LPP models extracted from Hugo [21]

[3] Table 3. Parameters of the driver model based on ISO 5982 [13] as adapted from Stein and Múčka [14]

[4] Table 4. Summary of NARX network computational efficiency for different numbers of input vectors and delays

\section{FIGURE CAPTIONS}

[1] Fig. 1. Identification procedure employed in the numerical verification.

[2] Fig. 2. A linear pitch-plane (LPP) vehicle model with a seated-driver model.

[3] Fig. 3. ISO PSD road roughness classification determined by Eq. (7).

[4] Fig. 4. A series-parallel architecture of the NARX model used for road profile reconstruction.

[5] Fig. 5. Vehicle responses to unit step road input for quarter car (QC) model displacements (a) and PSDs (c), sprung mass responses (solid) and unsprung mass 
responses (dotted), for LPP (b) and (d) with displacements of the rear suspension (dashed) and front suspension (dotted).

[6] Fig. 6. Actual (solid) vs. reconstructed profiles (dotted) for different road roughness classes.

[7] Fig. 7. Classification by ISO PSD and IRI (in $\mathrm{m}$ per $\mathrm{km}$ ) on the reconstructed profiles showing class boundaries (dashed), actual test road PSDs (dotted) and PSDs of reconstructed profiles (solid).

[8] Fig. 8. Reconstruction at different vehicle speeds: (a) $20 \mathrm{~km} / \mathrm{h}$, (b) $30 \mathrm{~km} / \mathrm{h}$, (c) 50 $\mathrm{km} / \mathrm{h}$, and (d) $80 \mathrm{~km} / \mathrm{h}$ with actual profiles (solid) and reconstructed profiles (dash-dot).

[9] Fig. 9. Network reconstructions at different speeds for three selected stages of road damage with actual profiles (dashed) over reconstructed profiles (dotted).

[10] Fig. 10. Identification under different vehicle speeds for growing defects, IRI values in $\mathrm{m}$ per $\mathrm{km}$.

[11] Fig. 11. Average correlation and error percentages for the different damage levels: $20 \mathrm{~km} / \mathrm{h}(\longrightarrow), 30 \mathrm{~km} / \mathrm{h}(\longrightarrow), 50 \mathrm{~km} / \mathrm{h}\left({ }^{-}\right)$and $80 \mathrm{~km} / \mathrm{h}(\longrightarrow)$.

[12] Fig.12. Average correlation coefficients and error percentages for each vehicle's speed. 
[13] Fig. 13. Displacement PSDs with IRI values for ride interpretation in $\mathrm{m}$ per $\mathrm{km}$.

[14] Fig. 14. Errors at each noise-signal ratio (SNR) averaged over 10 test runs when trained without noise.

[15] Fig. 15. ISO PSD classification at 0.2 SNR for different vehicle speeds, IRI values in $\mathrm{m}$ per $\mathrm{km}$.

[16] Fig.16. Reconstructed profiles in different payloads expressed as proportions of normal gross vehicle mass (GVM): (a) no added payload (b) 0.1 GVM (c) 0.2 GVM (d) 0.3 GVM (e) 0.4 GVM (f) 0.5 GVM.

[17] Fig. 17. ISO PSD classification for the different vehicle payloads in Fig. 16, IRI values in $\mathrm{n}$ per $\mathrm{km}$. 


\section{LIST OF TABLES}


Table 1. Static pre-load dependent parameters of the SDOF cushioned seat (from Wei and Griffin [12])

\begin{tabular}{lll}
\hline Pre-load & Damping Constant, & Spring Constant, \\
$(\mathrm{N})$ & $c_{0}(\mathrm{~N} \mathrm{~s} / \mathrm{m})$ & $k_{0}(\mathrm{~N} / \mathrm{m})$ \\
\hline 300 & 260 & 42300 \\
400 & 270 & 44121 \\
500 & 276 & 50210 \\
600 & 280 & 59300 \\
700 & 285 & 68000 \\
800 & 293 & 73000 \\
\hline
\end{tabular}


Table 2. Parameters of QC and LPP models extracted from Hugo [21]

\begin{tabular}{|c|c|c|c|c|c|c|c|c|c|c|}
\hline \multirow{2}{*}{$\begin{array}{l}\text { Model } \\
\text { QC }\end{array}$} & \multicolumn{4}{|c|}{ Mass $(\mathrm{kg})$ and Inertia $\left(\mathrm{kg} \mathrm{m}^{2}\right)$} & \multicolumn{2}{|c|}{$\begin{array}{l}\text { Damping } \\
\text { Constants } \\
(\mathrm{Ns} / \mathrm{m})\end{array}$} & \multicolumn{4}{|c|}{ Stiffness Constants $(\mathrm{N} / \mathrm{m})$} \\
\hline & $M_{S}$ & & $m_{u}$ & & $c_{S}$ & & $k_{s}$ & & $k_{t}$ & \\
\hline & 32895 & & 8578 & & $40 \times 10^{3}$ & & $2.6 \times 10^{6}$ & & $2.7 \times 10^{6}$ & \\
\hline \multirow[t]{2}{*}{ LPP } & $M_{s}$ & $\mathrm{I}_{\mathrm{s}}$ & $m_{u f}$ & $m_{u r}$ & $c_{s f}$ & $c_{s r}$ & $k_{s f}$ & $k_{s r}$ & $k_{t f}$ & $k_{t r}$ \\
\hline & 65790 & 263160 & 8578 & 8578 & $40 \times 10^{3}$ & 46152 & $2.6 \times 10^{6}$ & $3 \times 10^{6}$ & $2.7 \times 10^{6}$ & $5.4 \times 10^{6}$ \\
\hline
\end{tabular}


Table 3. Parameters of the driver model based on ISO 5982 [13] as adapted from Stein and Múčka [14]

\begin{tabular}{llll}
\hline & $m_{i}$ & $c_{i}$ & $k_{i}$ \\
$i$ & $\mathrm{~kg}$ & $(\mathrm{Ns} / \mathrm{m})$ & $(\mathrm{N} / \mathrm{m})$ \\
\hline 0 & 2 & --- & --- \\
1 & 6 & 387 & 9999 \\
2 & 2 & 234 & 34400 \\
3 & 42.5 & 1390 & 36200 \\
\hline
\end{tabular}


Table 4. Summary of NARX network computational efficiency for different numbers of input vectors and delays

\begin{tabular}{lllllll}
\hline $\begin{array}{l}\text { Inputs } \\
\text { (Fig. 2) }\end{array}$ & $\begin{array}{l}\text { No. } \\
\text { Delays }\end{array}$ & $\begin{array}{l}\text { ENOP } \\
\text { n/N }\end{array}$ & $\begin{array}{l}\text { SSE } \\
(\%)\end{array}$ & $\begin{array}{l}\text { SSW } \\
(\%)\end{array}$ & $\begin{array}{l}\text { Overall } \\
\text { Corr. } \\
(\%)\end{array}$ & $\begin{array}{l}\text { Duration } \\
(\mathbf{s})\end{array}$ \\
\hline$\ddot{z}_{s}$ & 8 & $60 / 361$ & 11.68 & 8.37 & 97.76 & 713 \\
$\ddot{z}_{s}$ & 15 & $116 / 641$ & 11.04 & 9.52 & 97.52 & 1418 \\
$\ddot{z}_{u f}$ & 15 & $121 / 641$ & 10.97 & 11.39 & 97.89 & 1410 \\
$\left\{\ddot{z}_{s}, \ddot{z}_{u f}\right\}^{T}$ & 15 & $151 / 941$ & 10.50 & 10.93 & 96.96 & 2735 \\
$\left\{\ddot{z}_{s}, \ddot{z}_{u f}, \ddot{z}_{1}\right\}^{T}$ & 8 & $95 / 681$ & 11.20 & 10.76 & 97.23 & 1569 \\
$\left\{\ddot{z}_{s}, \ddot{z}_{u f}, \ddot{z}_{1}\right\}^{T}$ & 15 & $163 / 1241$ & 10.37 & 11.16 & 96.73 & 4655 \\
\hline & & & & & & \\
\hline
\end{tabular}

ENOP - Effective number of training parameters represented by $\mathrm{n}$ while $\mathrm{N}$ is the total number of training parameters available network

SSE - sum of squared errors

SSW - sum of squared weights

Corr. - Correlation 


\section{LIST OF FIGURES}




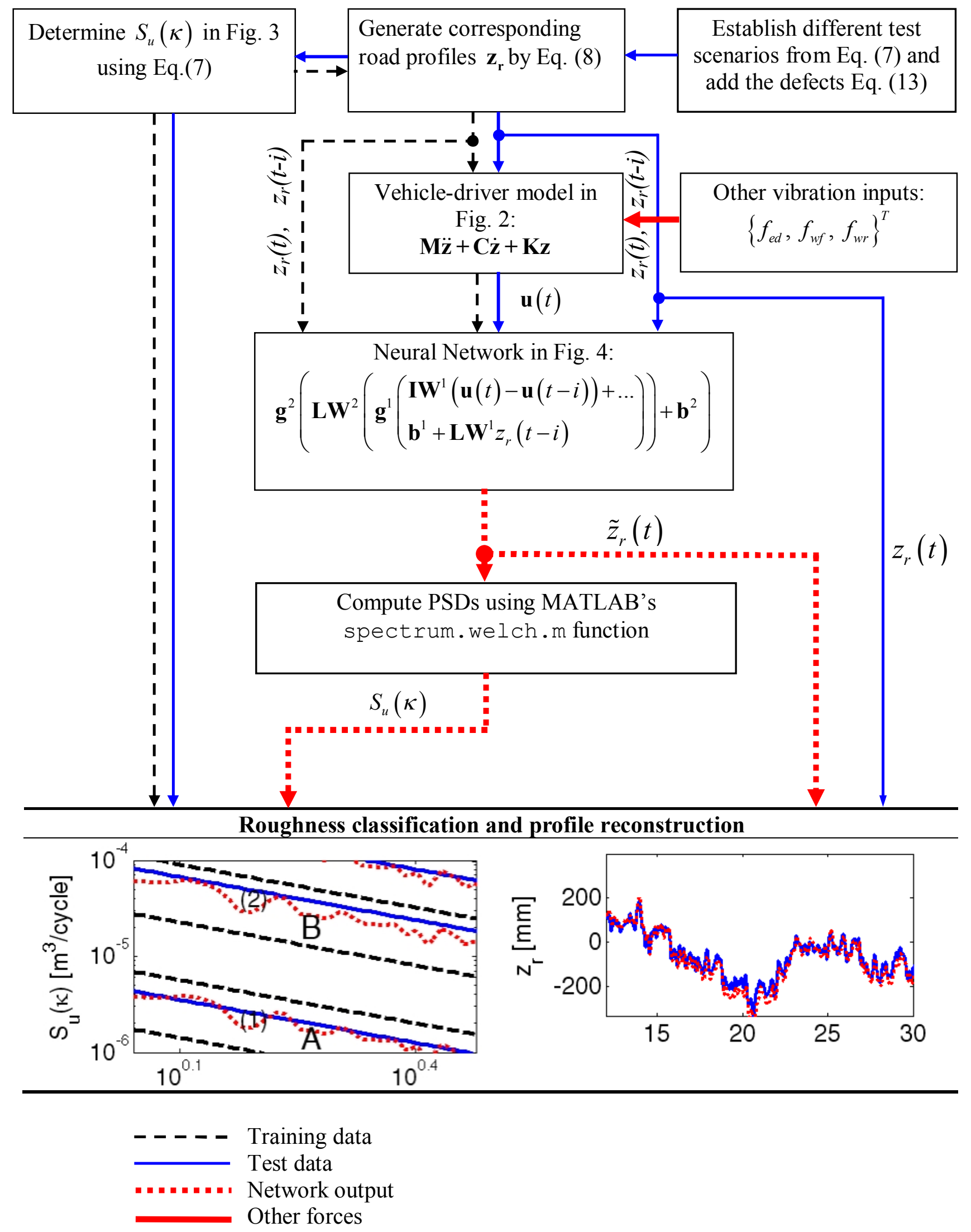

Fig. 1. Identification procedure employed in the numerical verification. 


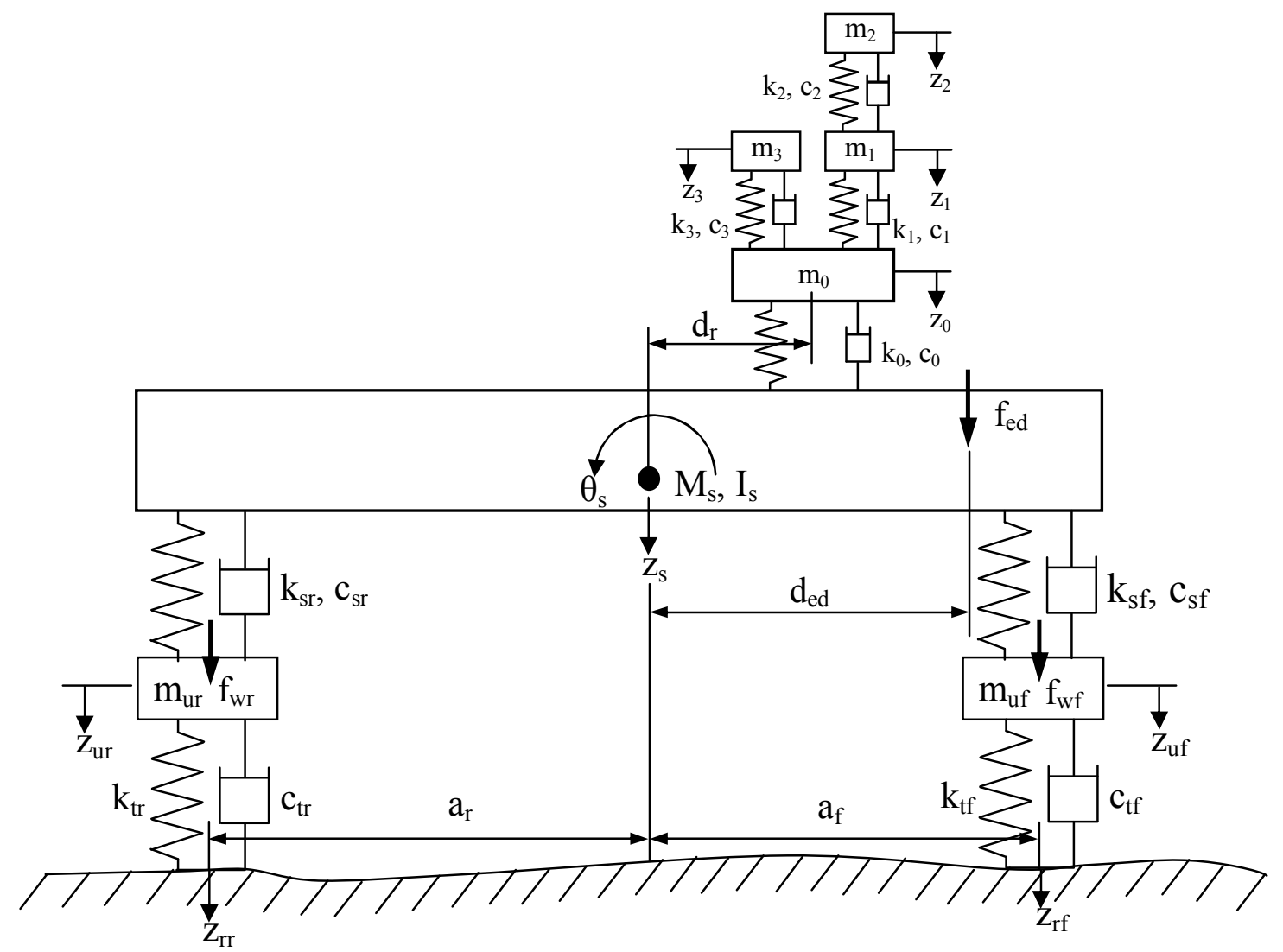

Fig. 2. A linear pitch-plane (LPP) vehicle model with a seated-driver model. 


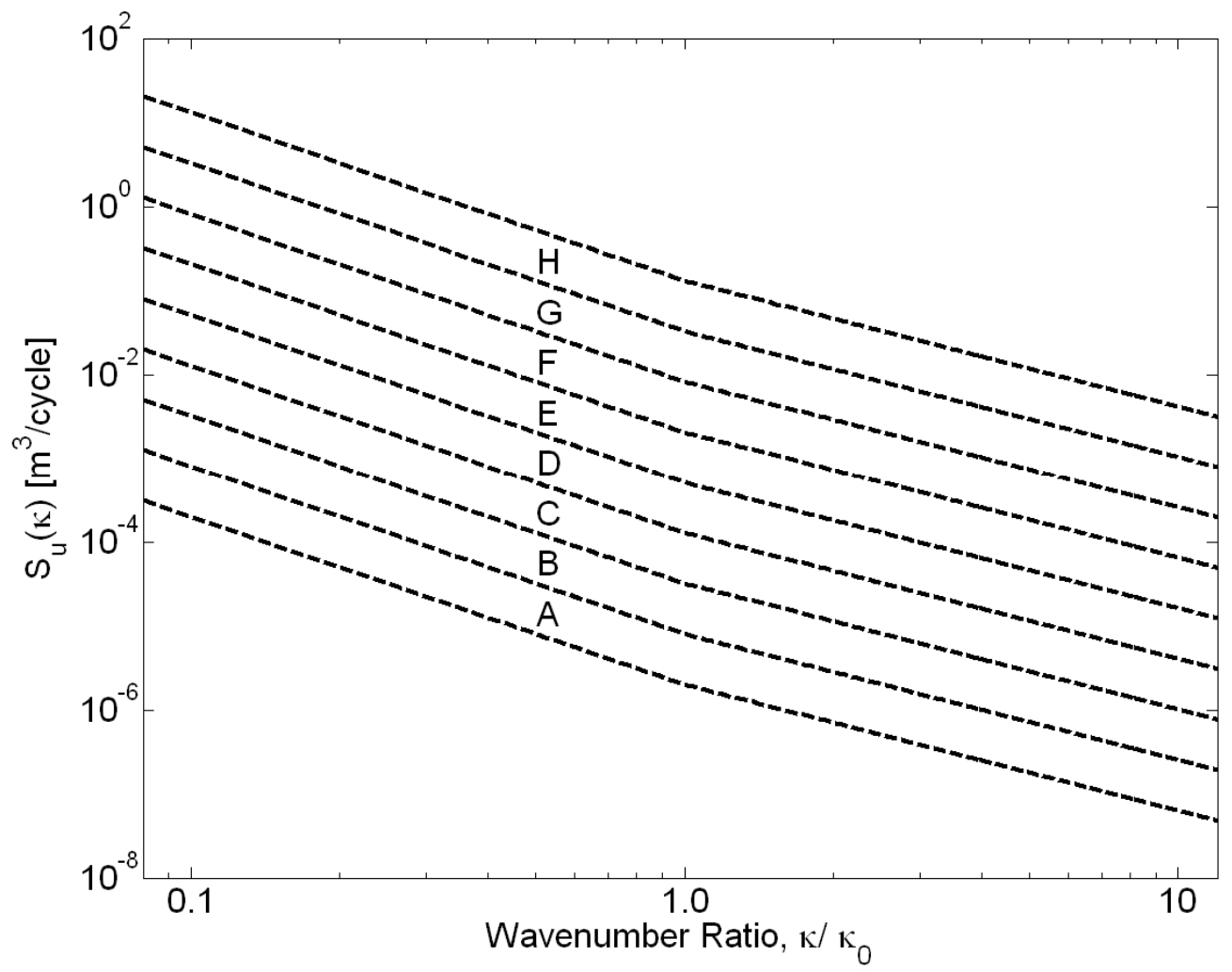

Fig. 3. ISO PSD road roughness classification calculated by Eq. 7. 


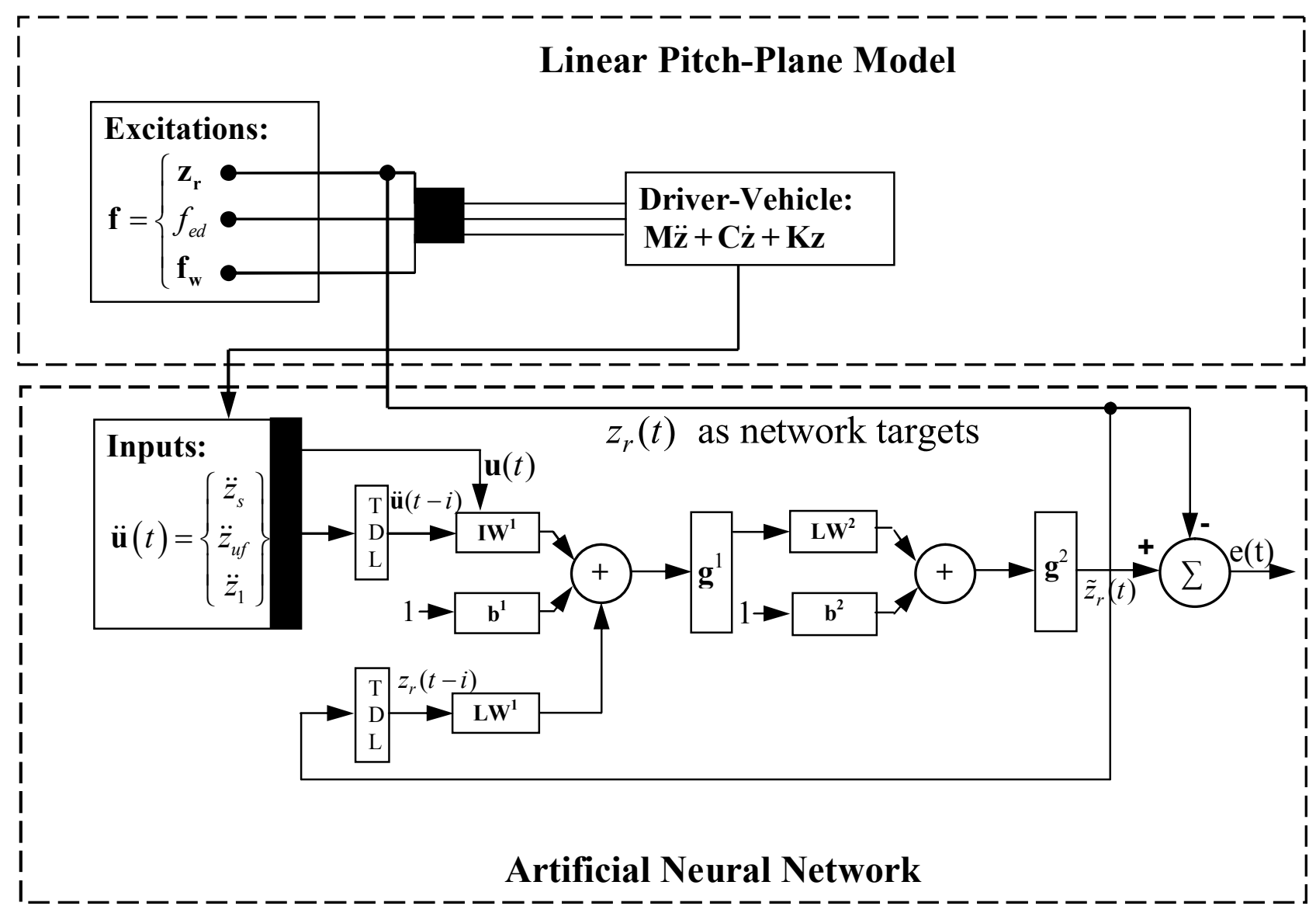

b: biases; IW: input weights; LW: layer weights and TDL: time delay lines

Fig. 4. A series-parallel architecture of the NARX model used for road profile reconstruction. 

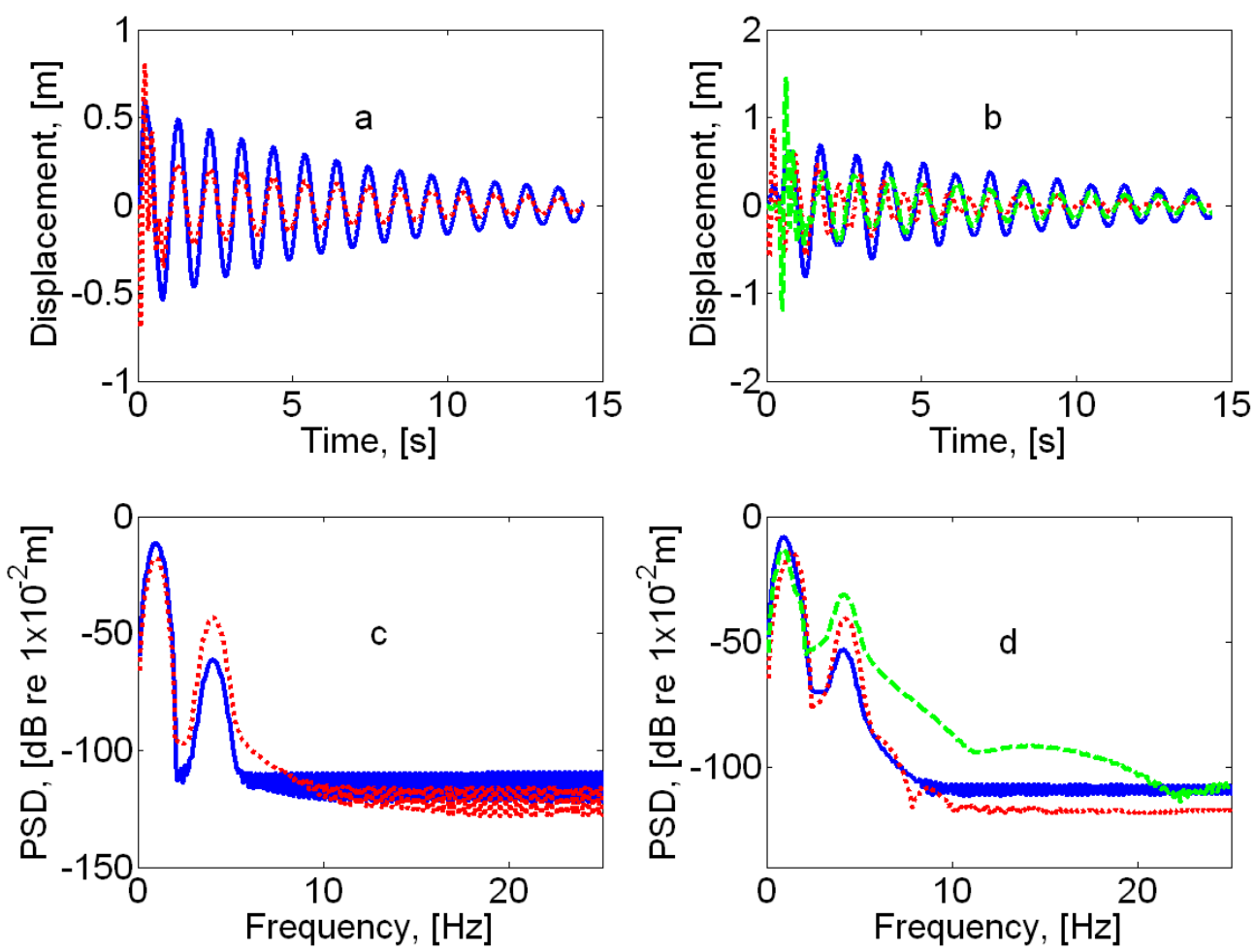

Fig. 5. Vehicle responses to unit step road input for quarter car (QC) model displacements (a) and PSDs (c), sprung mass responses (solid) and unsprung mass responses (dotted), for LPP (b) and (d) with displacements of the rear suspension (dashed) and front suspension (dotted). 

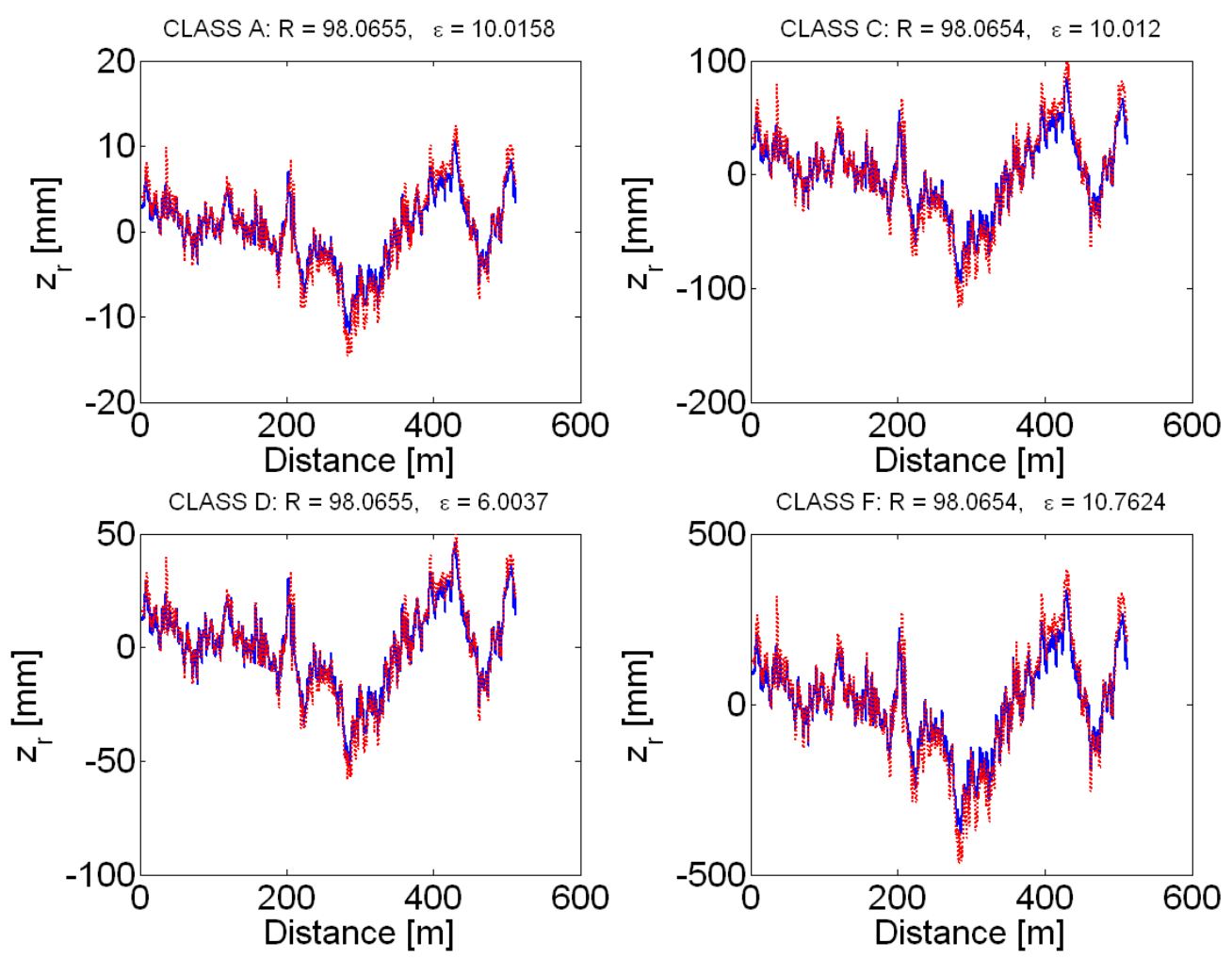

Fig. 6. Actual (solid) vs. reconstructed profiles (dotted) for different road roughness classes. 


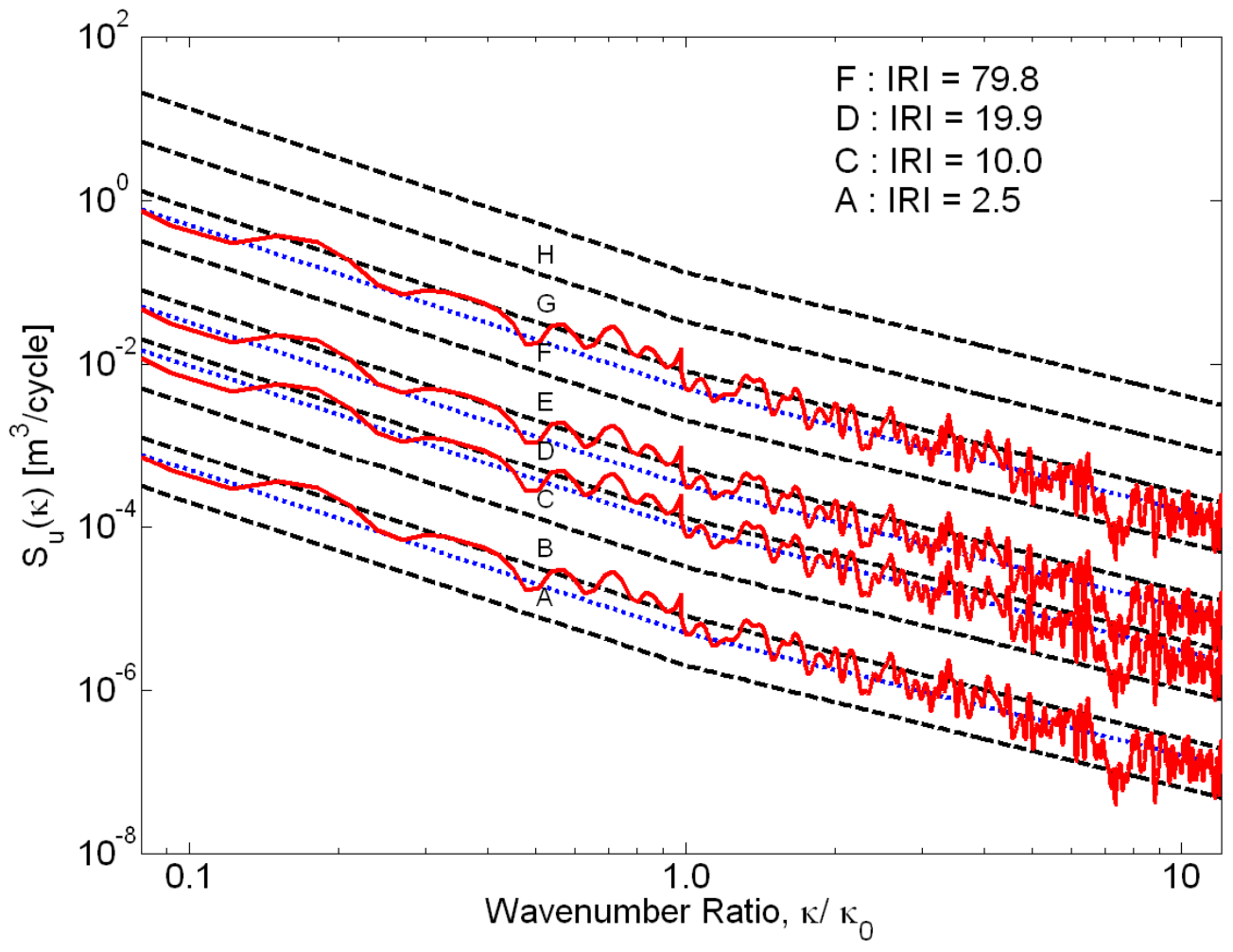

Fig. 7. Classification by ISO PSD and IRI (in $\mathbf{m}$ per $\mathrm{km}$ ) on the reconstructed profiles showing class boundaries (dashed), actual test road PSDs (dotted) and PSDs of reconstructed profiles (solid). 

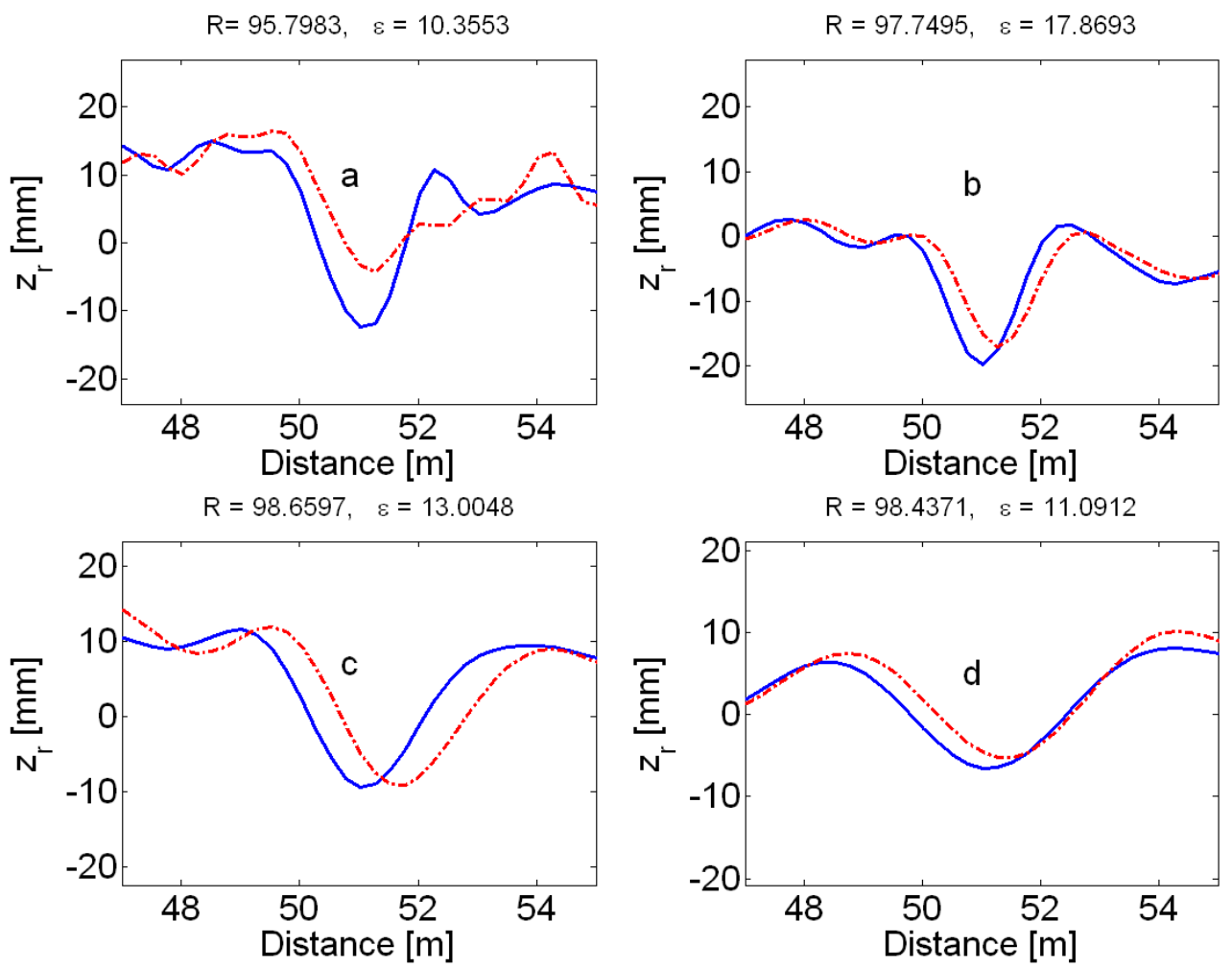

Fig. 8. Reconstruction at different vehicle speeds: (a) $20 \mathrm{~km} / \mathrm{h}$, (b) $30 \mathrm{~km} / \mathrm{h}$, (c) 50 $\mathrm{km} / \mathrm{h}$, and (d) $80 \mathrm{~km} / \mathrm{h}$ with actual profiles (solid) and reconstructed profiles (dashdot). 

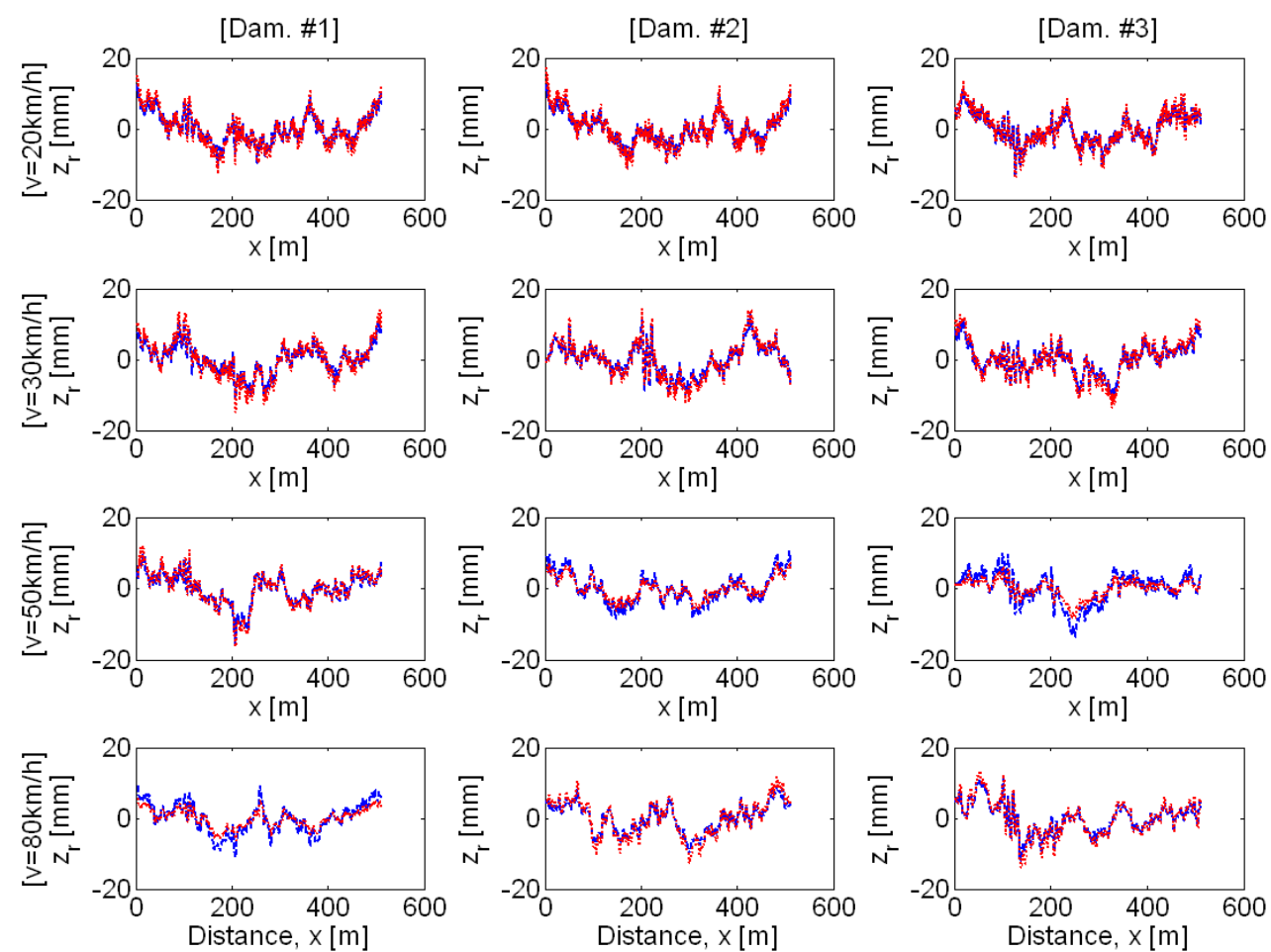

Fig. 9. Network reconstructions at different speeds for three selected stages of road damage with actual profiles (dashed) over reconstructed profiles (dotted). 



Fig. 10. Identification under different vehicle speeds for growing defects, IRI values in $\mathbf{m}$ per $\mathbf{k m}$. 

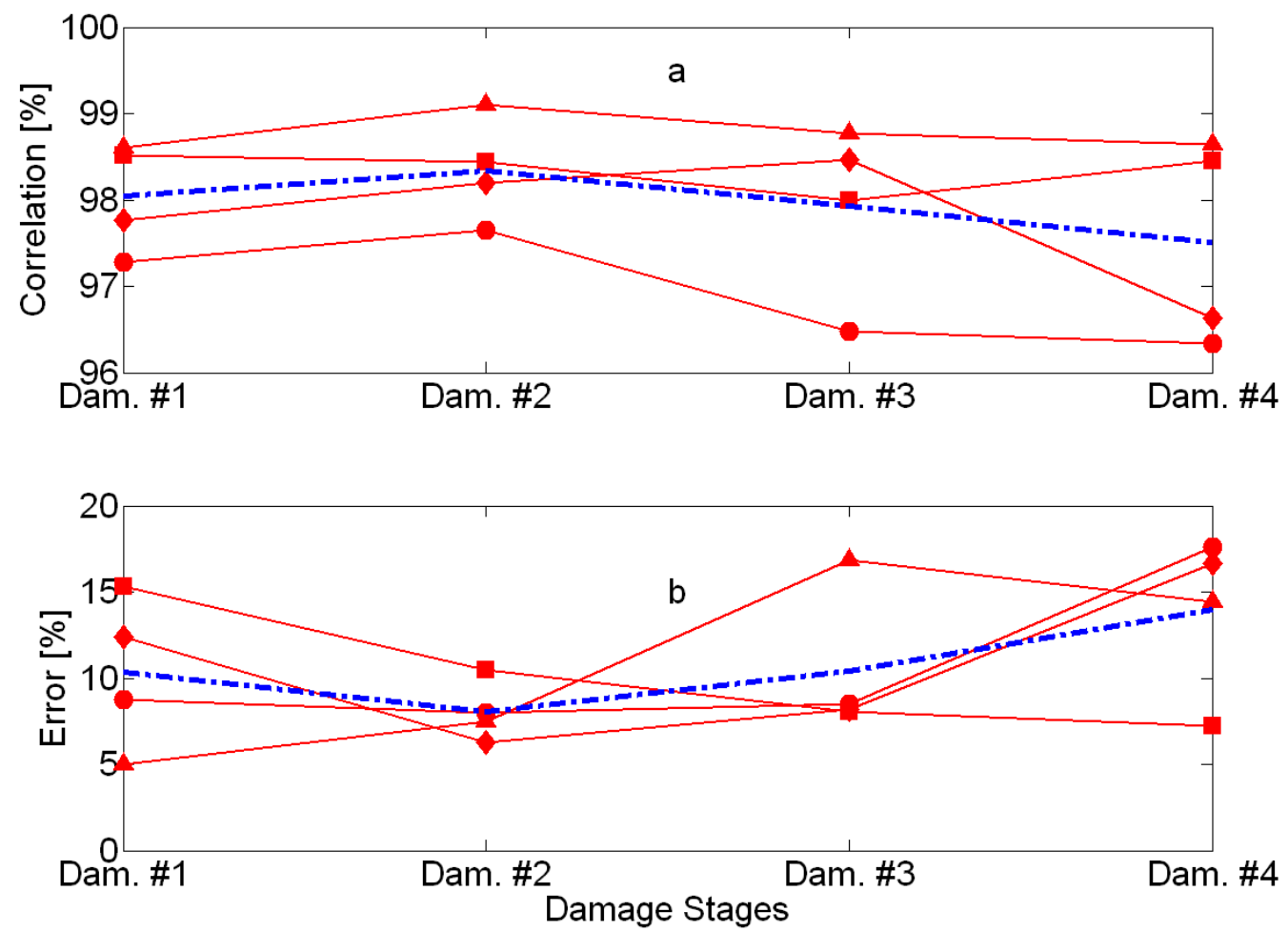

Fig. 11. Average correlation and error percentages for the different damage levels: $20 \mathrm{~km} / \mathrm{h}(\longrightarrow), 30 \mathrm{~km} / \mathrm{h}(\longrightarrow), 50 \mathrm{~km} / \mathrm{h}(\longrightarrow)$ and $80 \mathrm{~km} / \mathrm{h}(\longrightarrow)$ 

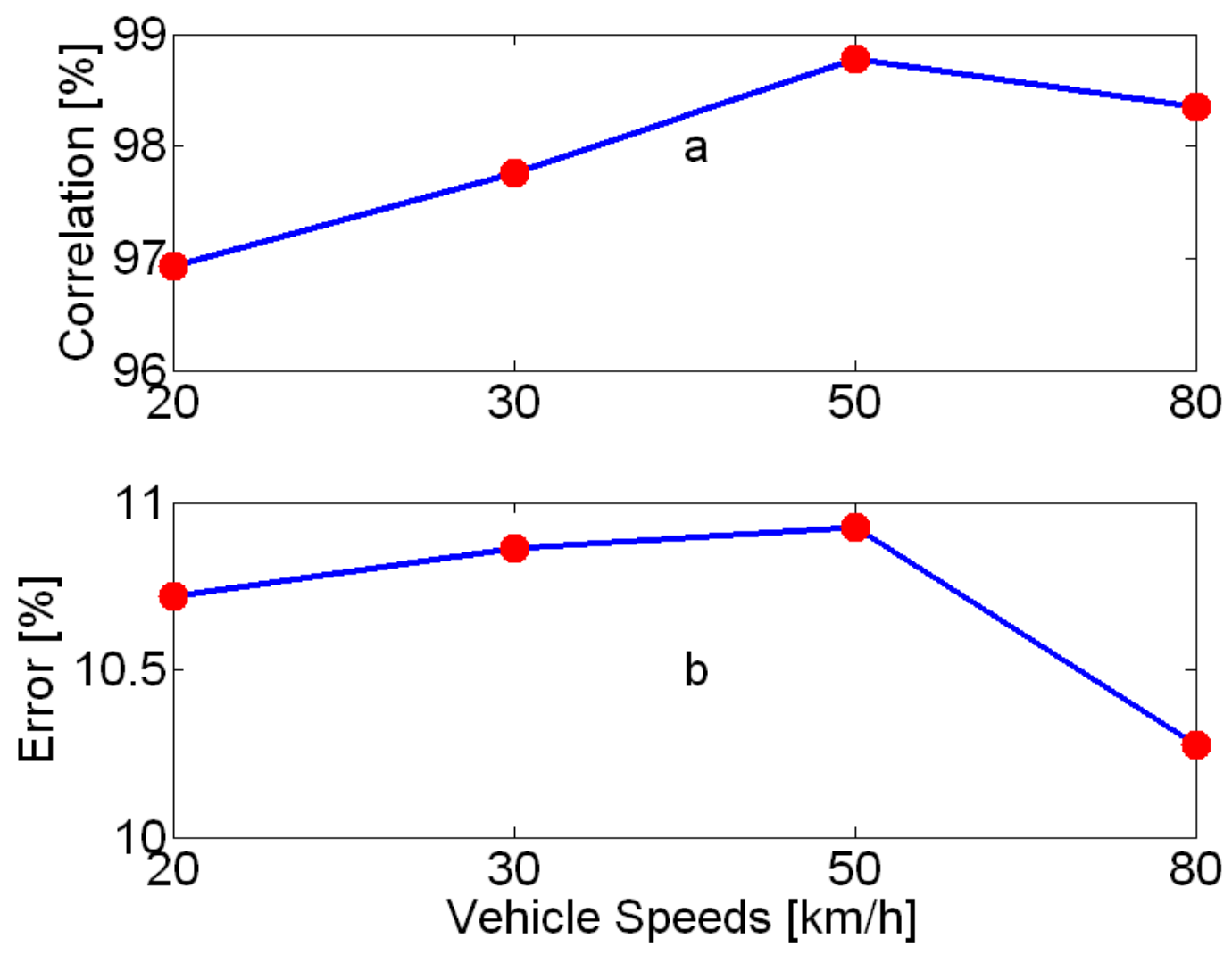

Fig. 12. Average correlation coefficients and error percentages for each vehicle's speed. 


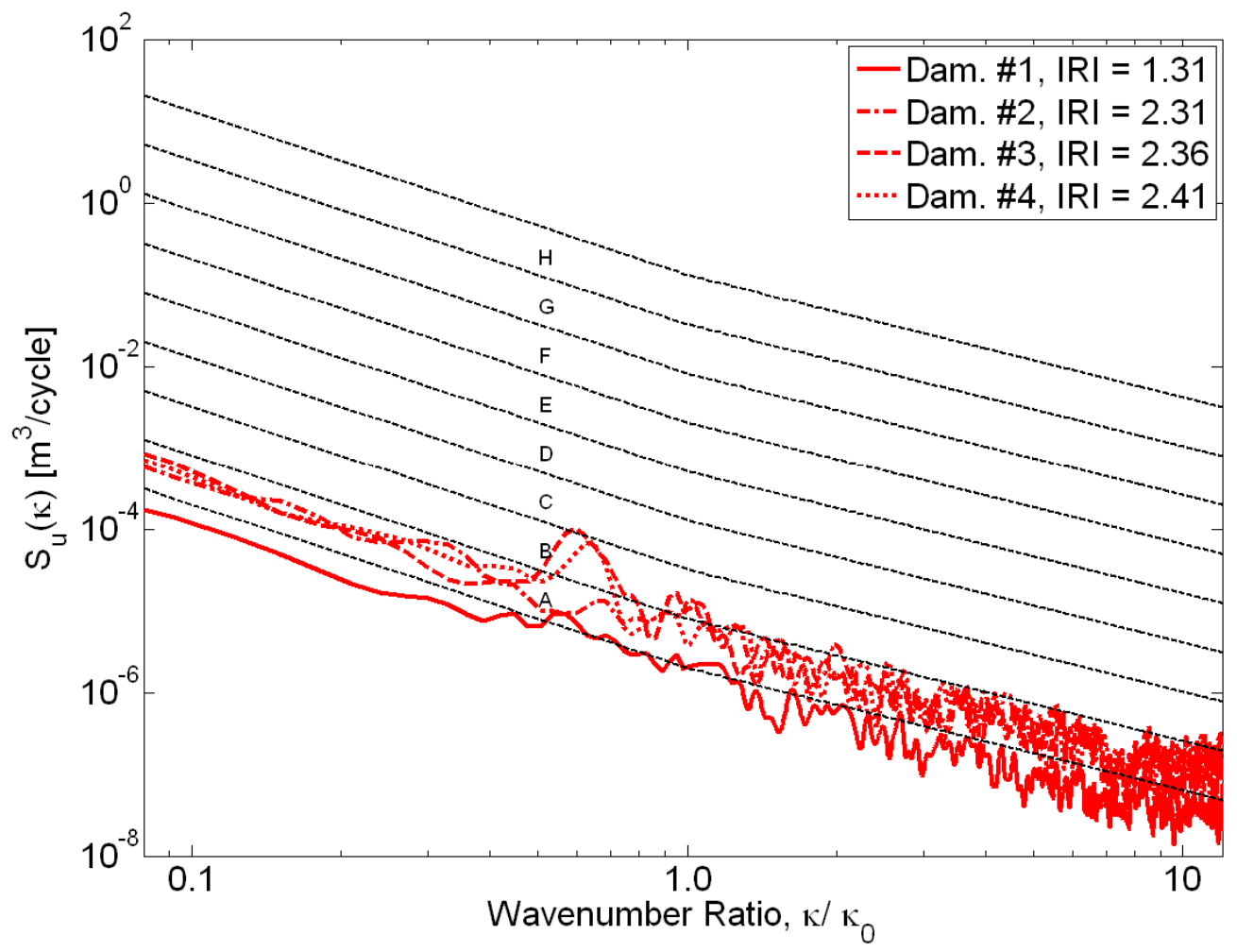

Fig. 13. Displacement PSDs with IRI values for ride interpretation in $\mathbf{m}$ per $\mathbf{k m}$. 


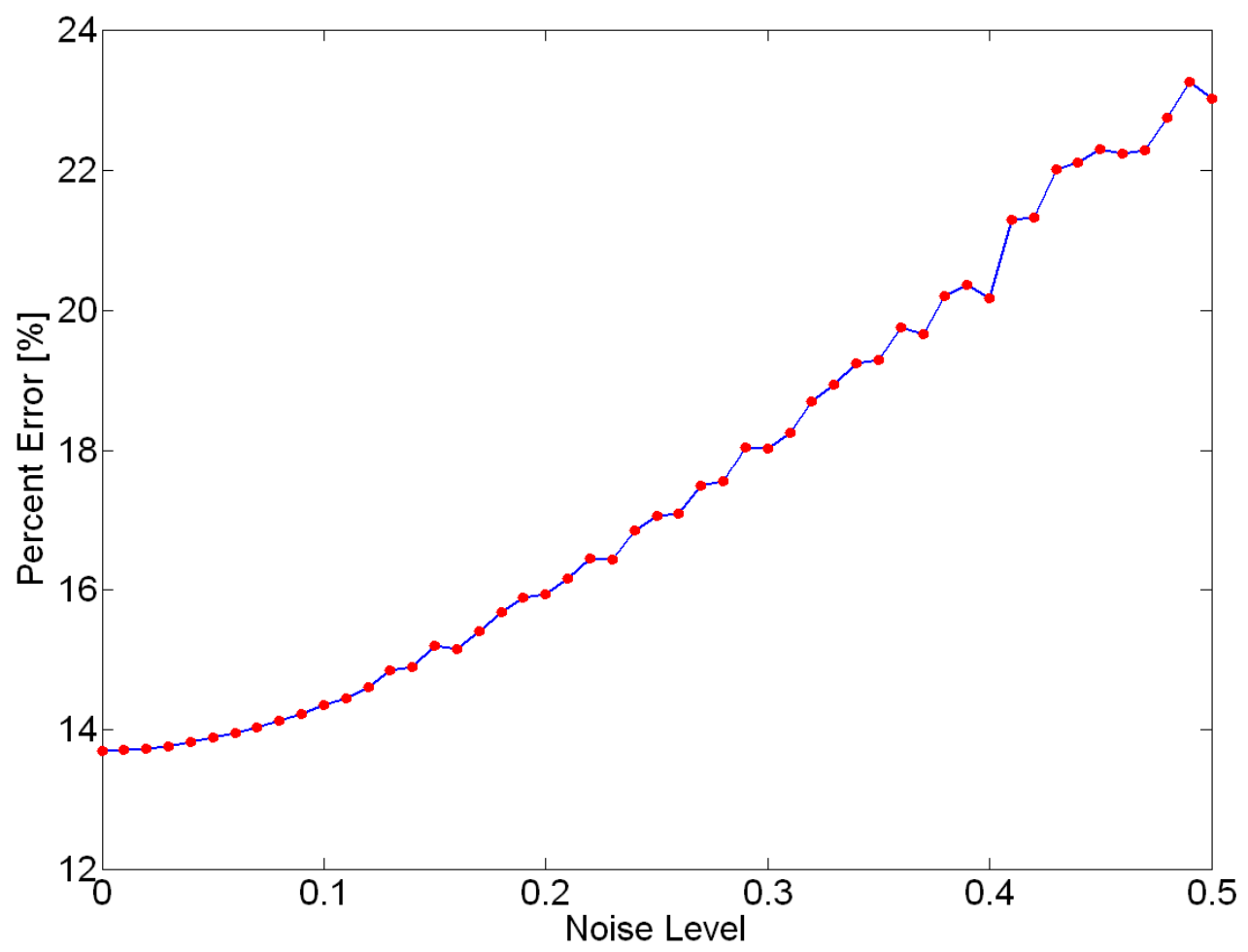

Fig. 14. Errors at each noise-signal ratio (SNR) averaged over 10 test runs when trained without noise. 


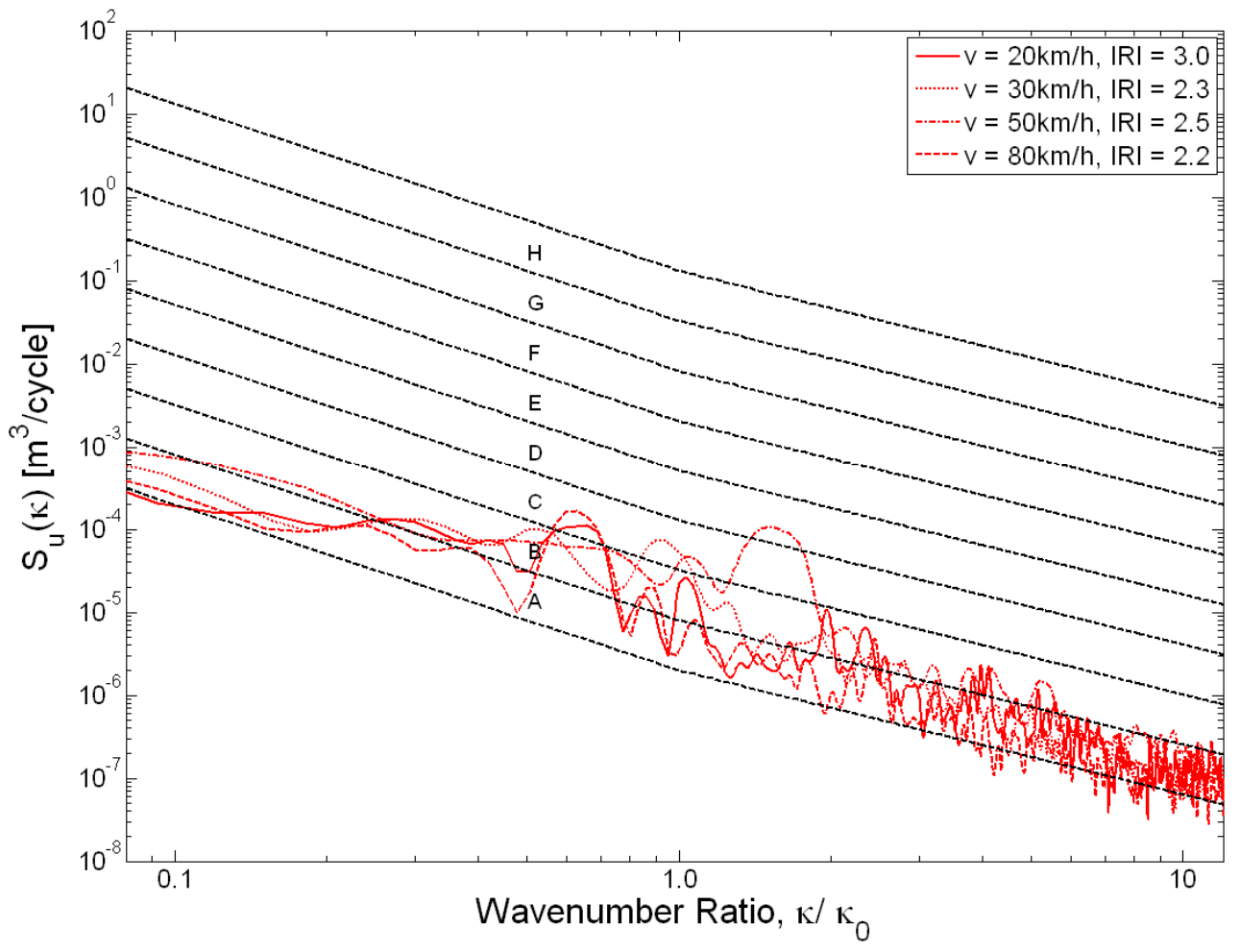

Fig. 15. ISO PSD classification at 0.2 SNR for different vehicle speeds, IRI values in $\mathbf{m}$ per $\mathbf{k m}$. 



Fig. 16. Reconstructed profiles in different payloads expressed as proportions of normal gross vehicle mass (GVM): (a) no added payload (b) 0.1 GVM (c) 0.2 GVM (d) 0.3 GVM (e) 0.4 GVM (f) 0.5 GVM. 


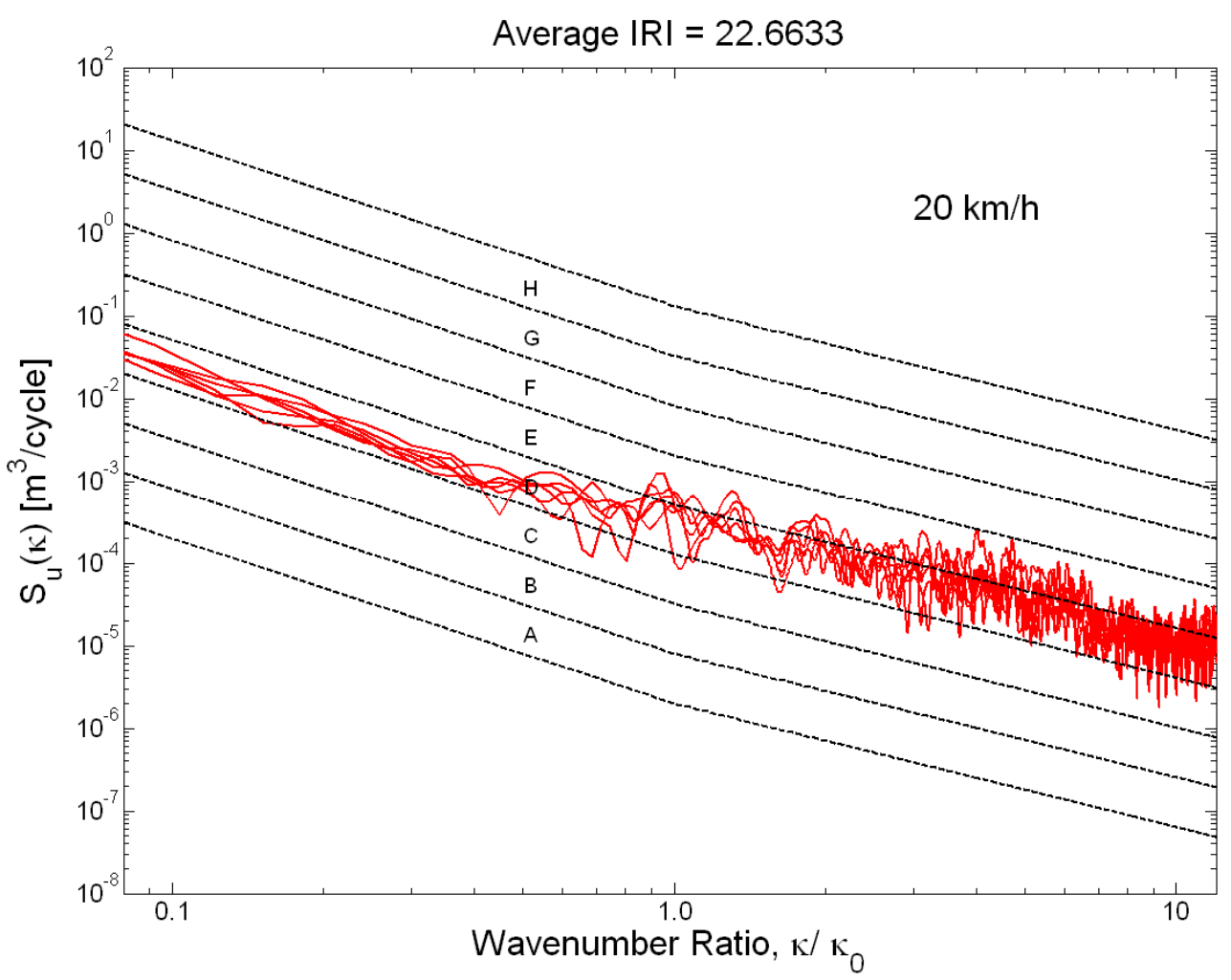

Fig. 17. ISO PSD classification for the different vehicle payloads in Fig. 16, IRI values in $\mathbf{m}$ per $\mathbf{k m}$. 